\title{
Hippo signaling interactions with Wnt/ $\beta$-catenin and Notch signaling repress liver tumorigenesis
}

\author{
Wantae Kim, ${ }^{1,2}$ Sanjoy Kumar Khan, ${ }^{1,2}$ Jelena Gvozdenovic-Jeremic, ${ }^{1}$ Youngeun Kim, ${ }^{3}$ Jason DahIman, ${ }^{1}$ Hanjun Kim, ${ }^{1,2}$ \\ Ogyi Park, ${ }^{4}$ Tohru Ishitani, ${ }^{5}$ Eek-hoon Jho, ${ }^{3}$ Bin Gao, ${ }^{4}$ and Yingzi Yang ${ }^{1,2}$ \\ 'Cenetic Disease Research Branch, National Human Genome Research Institute (NHGRI), NIH, Bethesda, Maryland, USA. ²Department of Developmental Biology, Harvard School of Dental Medicine (HSDM), \\ Boston, Massachusetts, USA. ${ }^{3}$ Department of Life Sciences, University of Seoul, Seoul, South Korea. ${ }^{4}$ Section on Liver Biology, National Institute on Alcohol Abuse and Alcoholism (NIAAA), NIH, \\ Bethesda, Maryland, USA. ${ }^{5}$ Division of Cell Regulation Systems, Medical Institute of Bioregulation, Kyushu University, Fukuoka, Japan.
}

\begin{abstract}
Malignant tumors develop through multiple steps of initiation and progression, and tumor initiation is of singular importance in tumor prevention, diagnosis, and treatment. However, the molecular mechanism whereby a signaling network of interacting pathways restrains proliferation in normal cells and prevents tumor initiation is still poorly understood. Here, we have reported that the Hippo, Wnt/ $\beta$-catenin, and Notch pathways form an interacting network to maintain liver size and suppress hepatocellular carcinoma (HCC). Ablation of the mammalian Hippo kinases Mst1 and Mst2 in liver led to rapid HCC formation and activated Yes-associated protein/WW domain containing transcription regulator 1 (YAP/TAZ), STAT3, Wnt/ $\beta$-catenin, and Notch signaling. Previous work has shown that abnormal activation of these downstream pathways can lead to HCC. Rigorous genetic experiments revealed that Notch signaling forms a positive feedback loop with the Hippo signaling effector YAP/TAZ to promote severe hepatomegaly and rapid HCC initiation and progression. Surprisingly, we found that Wnt/קcatenin signaling activation suppressed HCC formation by inhibiting the positive feedback loop between YAP/TAZ and Notch signaling. Furthermore, we found that STAT3 in hepatocytes is dispensable for HCC formation when mammalian sterile 20-like kinase 1 and 2 (Mst1 and Mst2) were removed. The molecular network we have identified provides insights into HCC molecular classifications and therapeutic developments for the treatment of liver tumors caused by distinct genetic mutations.
\end{abstract}

\section{Introduction}

Hepatocyte proliferation and survival are tightly controlled by many signaling pathways. The Hippo, STAT3, Wnt, and Notch pathways are evolutionarily conserved and critically regulate cell proliferation, fate determination, and survival. In the liver, misregulation of these signaling pathways led to hepatocellular carcinoma (HCC) in human patients and animal models (1-6). HCC is the fifth most common cancer and second leading cause of cancer deaths in the world $(6,7)$. It is imperative to understand how these signaling pathways interact with each other when causing HCC, as the same mutations in one signaling pathway are likely to have distinct effects on the disease, depending on the genetic status of its interacting pathways $(2,8)$.

In mammals, the Hippo pathway is a signaling cascade containing mammalian sterile 20-like kinase 1 and 2 (MST1/2) kinases, the scaffold protein Salvador, large tumor suppressor kinases 1 and 2 (LATS1/2), and the transcription factors Yes-associated protein and WW domain containing transcription regulator 1 (YAP/TAZ) (9-12). This pathway can be activated by cell density, mechanical cues, and GPCR signaling (13-16), which eventually leads to phosphorylation of the YAP/TAZ transcription factors (17-19). Phosphorylated YAP and TAZ are retained in the cytoplasm and degraded by a $\beta$-TrCPmediated proteasomal pathway. Unphosphorylated YAP and TAZ

Conflict of interest: The authors have declared that no conflict of interest exists. Submitted: May 9, 2016; Accepted: October 13, 2016.

Reference information: / Clin Invest. 2017;127(1):137-152. doi:10.1172/JCI88486. enter the nucleus to activate gene expression that promotes cell proliferation and survival. The Hippo signaling pathway is tumor suppressive, as its inactivation leads to tissue overgrowth and tumor formation in a broad range of tissues (9, 20-23). Interestingly, Hippo-deficient liver exhibits more rapid cirrhosis and HCC development than do other mouse models of liver cancer $(24,25)$. $\beta$-Catenin is a centrally important transcriptional coactivator that activates Wnt target gene expression in the Wnt/ $\beta$-catenin pathway (26). Abnormal activation of the Wnt/ $\beta$-catenin pathway causes many types of tumors in humans including hepatoblastoma and HCC (27). In mice, hepatocyte-specific expression of active $\beta$-catenin resulted in hepatomegaly and HCC development when induced by the chemical carcinogen diethylnitrosamine (DEN) (27-30), and DEN injection is required in many genetic liver tumor models (1, 31). Importantly, HCC forms in $100 \%$ of $M s t 1 / 2$ double-mutant mouse livers by the age of 4 months, without chemical induction, while $50 \%$ of transgenic mice overexpressing TGF- $\alpha$ or E2F1 develop HCCs when older than 12 months $(32,33)$. These observations suggest that the Hippo signaling pathway is a critical gatekeeper that prevents liver tumor initiation. Notably, MST1/2 inhibition and the resultant augmentation of YAP/TAZ activity have been shown in approximately $30 \%$ of human HCCs.

Hippo signaling interacts with other signaling pathways that cause HCC when abnormally activated, highlighting the importance of understanding an interacting signaling network, rather than a single signaling pathway, in suppressing liver tumor formation. We found here that in the Hippo-deficient 
A

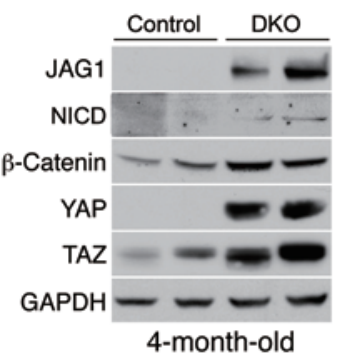

B

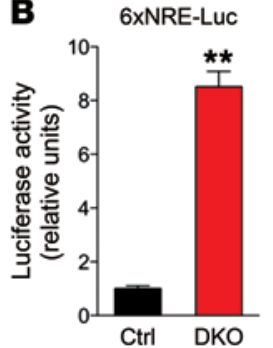

C

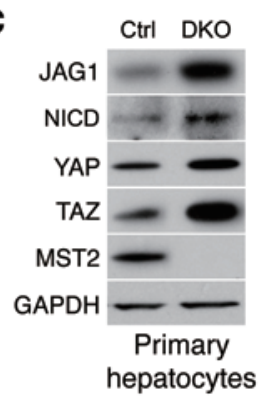

$\mathbf{F}$

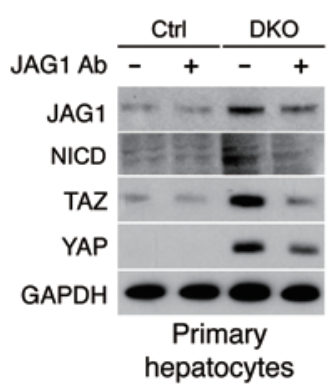

G

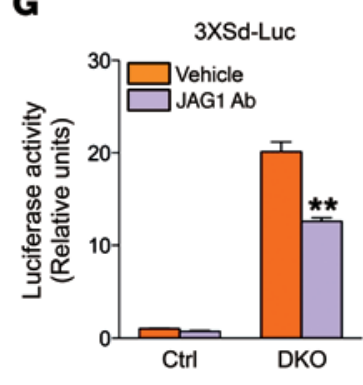

D

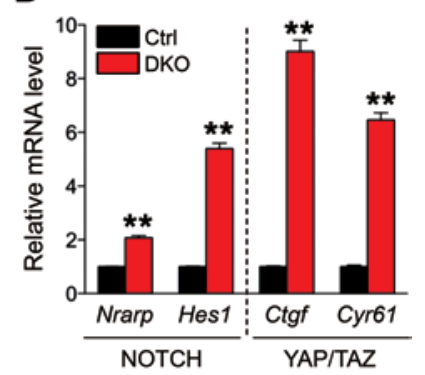

H

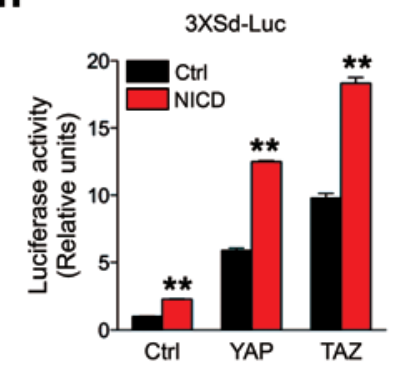

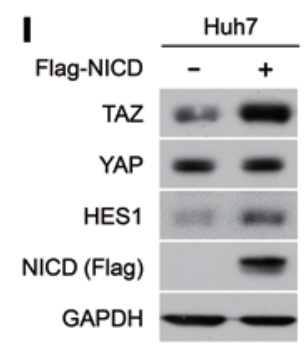
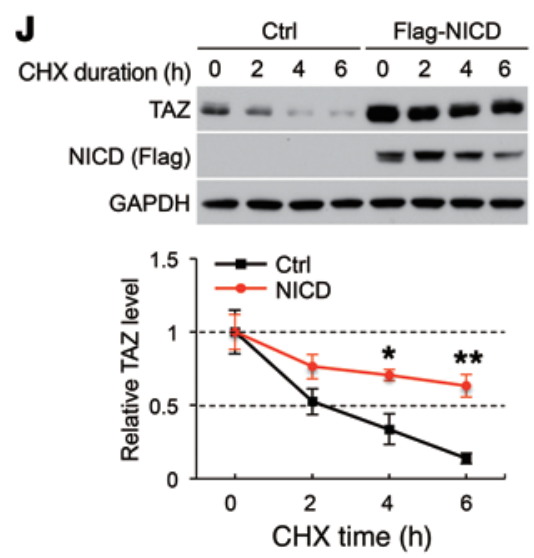

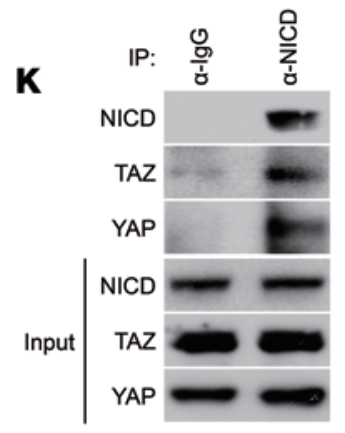

$\mathbf{L}$

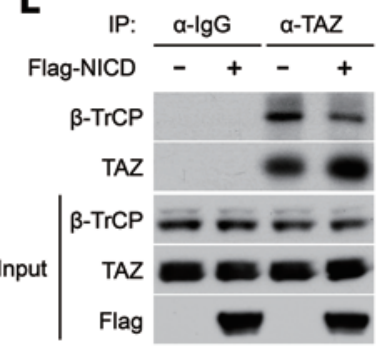

Figure 1. Notch signaling activates and forms a positive feedback loop with YAP/TAZ. (A) Western blot analysis of control and DKO liver tissues with the indicated antibodies. (B) Notch reporter assay in primary hepatocytes derived from control and DKO mice $(n=3)$. (C) Western blot analysis of JAG1, $\beta$-catenin, YAP, and TAZ protein levels in primary hepatocytes derived from control and DKO mice. (D) qRT-PCR of Notch or YAP/TAZ response gene expression in liver tissues from control and DKO mice $(n=3)$. (E) JAG1 upregulation in DKO liver depended on the presence of YAP or YAP/TAZ. Western blot analysis of lysates from the indicated mice. (F) Western blot analysis of primary hepatocytes treated with neutralizing anti-JAG1 antibody (20 $\mu$ /ml). (C) YAP/TAZ-dependent reporter assay in primary hepatocytes isolated from control and DKO-treated mice with $15 \mu \mathrm{g} / \mathrm{ml}$ neutralizing anti-JAC1 antibody $(n=3)$. (H) Increase in YAP/ TAZ reporter activity by NICD in Huh7 cells. (I) Western blot analysis of the indicated protein levels induced by NICD expression in Huh7 cells. (J) Western blot analysis of TAZ protein in Huh7 cells treated with $25 \mu \mathrm{g} / \mathrm{ml} \mathrm{CHX} \mathrm{for} \mathrm{the} \mathrm{indicated} \mathrm{durations.} \mathrm{Line} \mathrm{graphs} \mathrm{show} \mathrm{quantified} \mathrm{TAZ} \mathrm{levels} \mathrm{at} \mathrm{the} \mathrm{indicated} \mathrm{time}$ points $(n=4)$. Data in J represent the mean \pm SD. ${ }^{*} P<0.05$ and ${ }^{* *} P<0.01$, by 2-tailed Student's $t$ test. (K) NICD associated with endogenous TAZ or YAP in Huh7 cells. IP was performed using anti-NICD antibody, and coprecipitated protein was analyzed by Western blotting. (L) Flag-tagged NICD was transfected into Huh7 cells, which were treated with $20 \mu \mathrm{M}$ MG132 for 8 hours. IP with anti-TAZ antibody was performed to detect interaction with $\beta$-TrCP. (D, G, and H) Data are expressed as the mean \pm SEM. ${ }^{*} P<0.05$ and ${ }^{* *} P<0.01$, by 2 -tailed Student's $t$ test. Ctrl, control.

liver, Wnt/ $\beta$-catenin, Notch, and STAT3 signal were activated. Although each one of these downstream events can cause HCC $(27,34-38)$, we demonstrate with in vivo genetic approaches that they differentially contribute to the HCC formation caused by loss of Hippo signaling. While Notch signaling forms a positive feedback loop with YAP/TAZ to promote rapid tumor initiation in the Hippo-deficient liver, STAT3 activation is dispensable, and $\beta$-catenin inhibits tumor initiation by inhibiting Notch sig- naling. Therefore, the tumorigenic effects of a particular signaling pathway depend on the status of its interacting pathways.

\section{Results}

Notch signaling forms a positive feedback loop with YAP/TAZ. We and other groups have shown previously that inactivation of Hippo signaling in the liver leads to hepatomegaly and rapid tumor initiation and progression $(24,25,39,40)$. However, it is still large- 
A

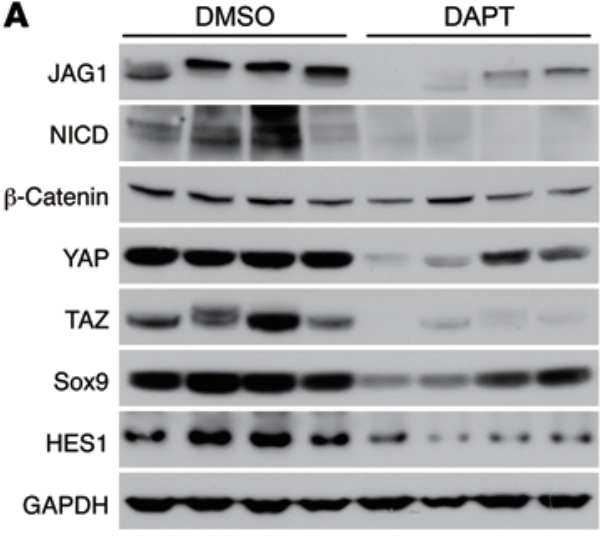

B

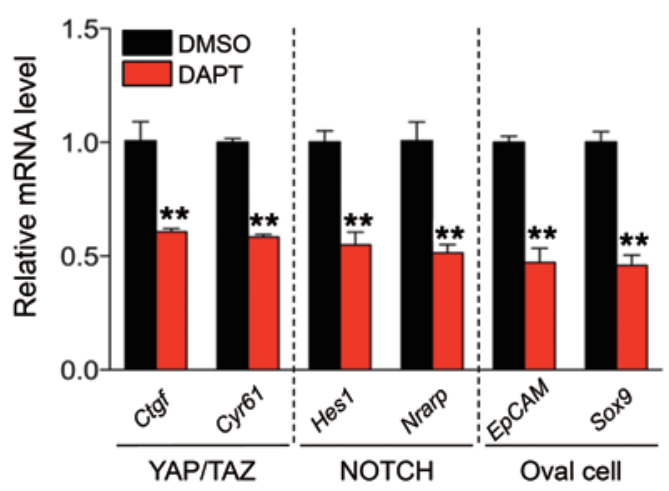

C

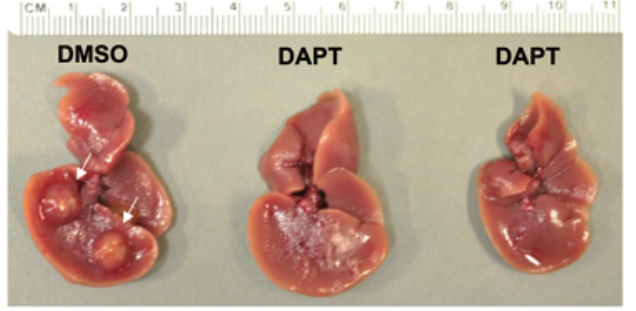

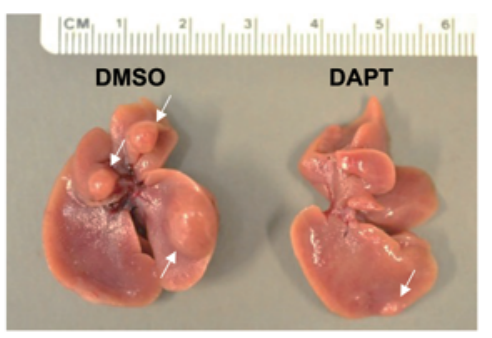

Figure 2. Pharmacological inhibition of Notch signaling suppresses YAP/TAZ activity in vivo. (A) DKO mice received 20 i.p. injections of DAPT at a concentration of $125 \mathrm{mg} /$ kg every other day or vehicle DMSO (control). Western blot analysis of lysates from DAPT- or DMSO-injected mouse livers. (B) qRT-PCR analysis showed significantly reduced expression of YAP/TAZ target genes, Notch-response genes, and oval cell markers in liver from DAPT-injected mice ( $n=4$ per group). (C) Representative macroscopic images of livers treated with DMSO or DAPT. White arrows indicate tumor nodules. (D-F) Quantitative analysis of liverto-BW ratio (D), tumor numbers (E), and maximal tumor size $(\mathbf{F})(n=7)$. Data are expressed as the mean \pm SEM. ${ }^{*} P<0.01$, by 2 -tailed Student's $t$ test. $P$ values in $\mathbf{D}-\mathbf{F}$ were determined by 2-tailed Student's $t$ test.
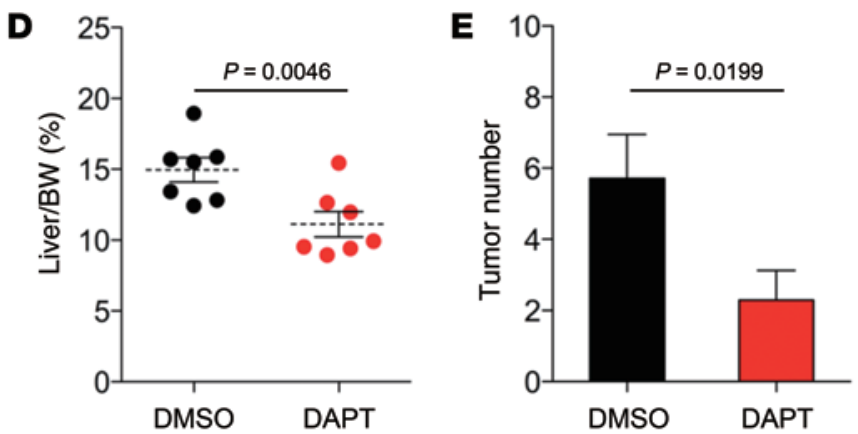

ly unknown whether inactivation of Hippo signaling causes liver tumor formation by misregulating other signaling pathway(s) that are critical for controlling hepatocyte proliferation and survival. Oval cells (liver progenitor/stem cells) and bile ductal cells both express Sox9 and EPCAM $(41,42)$. The marked expansion of oval/ductal cells observed in the albumin-Cre (Alb-Cre) $\mathrm{Mst1}^{-1}$ $M s t 2^{f l / f l}$ (referred to hereafter as DKO) mutant liver $(24,25,39)$ led us to hypothesize that Notch signaling may have been upregulated, as its activation promotes oval/ductal cell formation (37, $43,44)$. The Notch pathway is activated by direct cell-cell contact that allows direct binding of Notch receptors and ligands (jagged and Delta-like). Notch ligand binding induces sequential proteolytic cleavage of Notch receptors to generate Notch intracellular domain (NICD) (45), which enters the nucleus to participate in the transcriptional regulation of target genes (46-48). As with previous studies $(49,50)$, the expression of jagged 1 (Jag1), which encodes the Notch ligand JAG1, and Notch genes were upregulated in the DKO liver (Figure 1A and Supplemental Figure 1, A and $\mathrm{B}$; supplemental material available online with this article; doi:10.1172/JCI88486DS1). We then examined Notch signaling activities directly and found that Notch reporter activity, NICD

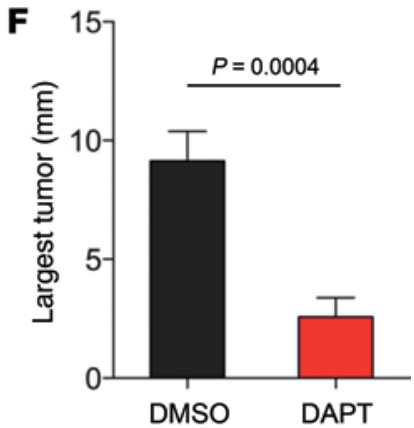

levels, and Notch target gene expression were significantly increased in the DKO liver and primary hepatocytes (Figure 1, B-D and Supplemental Figure 1C).

To test whether Jag1 activation is required to activate Notch signaling in the DKO liver, we applied the anti-JAG1 antibody to the DKO primary hepatocytes and found that Notch signaling was reduced (Supplemental Figure 1D), suggesting that JAG1 upregulation is important in activating Notch signaling in the DKO liver. Furthermore, we found that upregulation of Jag1 expression and Notch signaling in the DKO liver was Yap and Taz dependent. Partial removal of Yap or Yap and Taz led to reduced Jag1 expression in the mouse liver in vivo (Figure 1E), and in vitro, knocking down YAP or TAZ led to reduced Notch signaling reporter activity in the HCC cell line Huh7 (Supplemental Figure $1, \mathrm{E}$ and $\mathrm{F}$ ). In addition, we found that overexpressing YAP or TAZ in Huh7 cells led to upregulated JAG1 expression and Notch signaling activity (Supplemental Figure 1, G and H). These results indicate that the Hippo kinases MST1 and MST2 inhibit Notch signaling by inhibiting the expression of Jag1 that is activated by YAP and TAZ.

Interestingly, in DKO hepatocytes treated with the JAG1 antibody, we also found that the levels of TAZ and YAP protein as well as JAG1 protein itself were reduced (Figure $1 \mathrm{~F}$ ). In addition, 
A

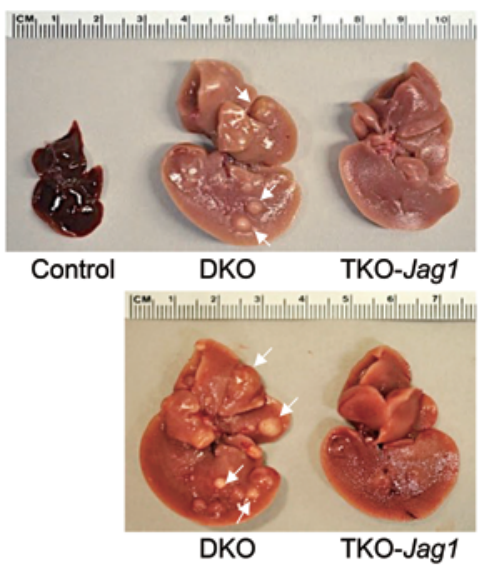

B

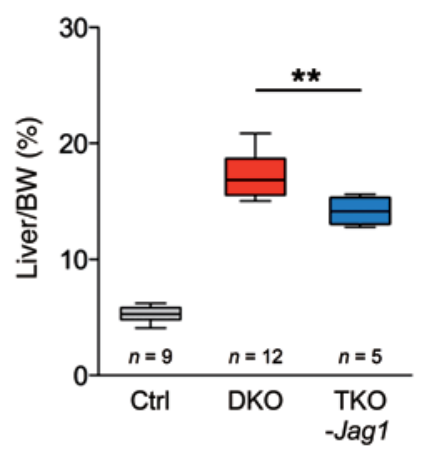

C

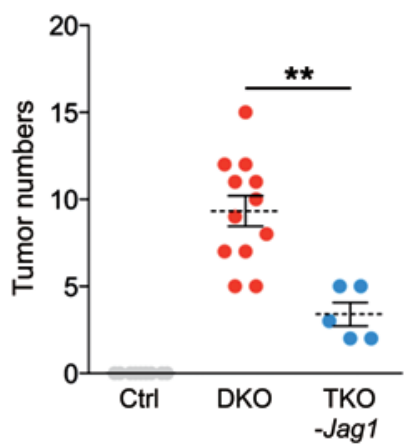

D

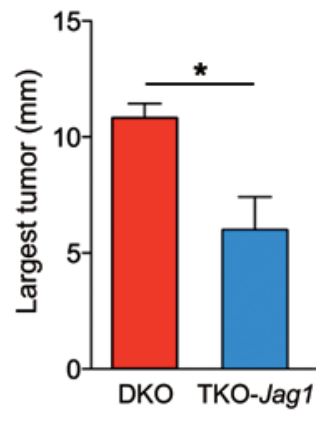

E

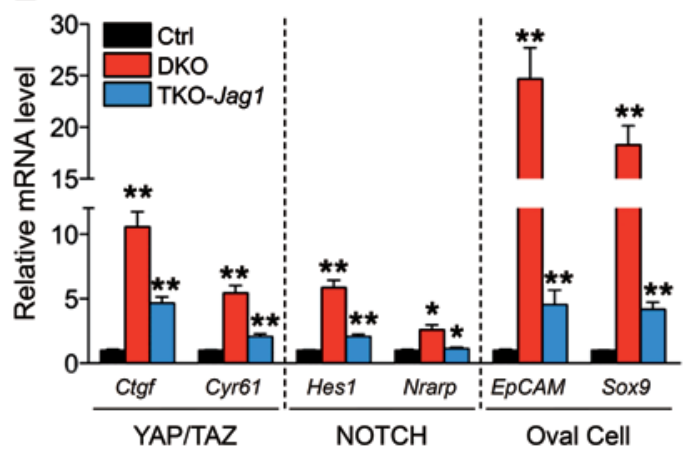

G

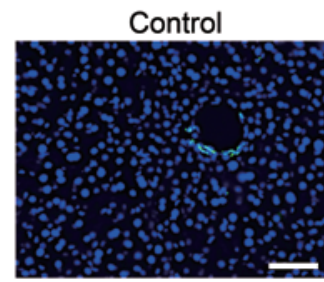

TKO-Jag1

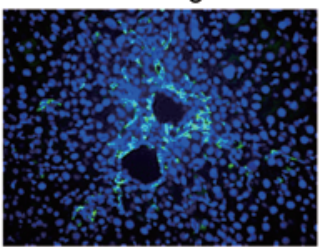

DKO

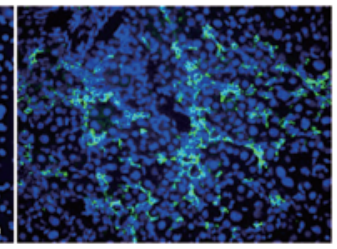

Epcam/DAPI
$\mathbf{F}$

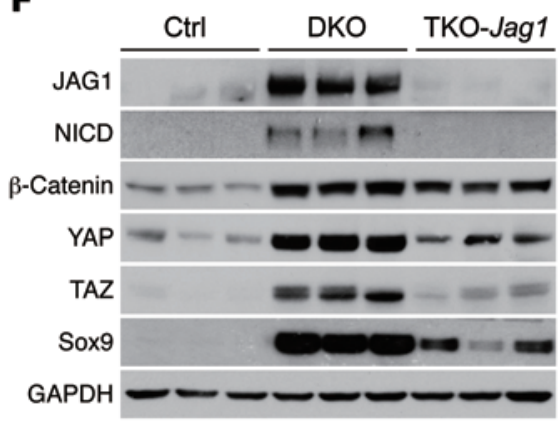

Notch signaling inhibition by treatment with DAPT $(N-[(3,5-$ difluorophenyl)acetyl]-L-alanyl-2-phenyl]glycine-1,1-dimethylethyl ester), an inhibitor of the $\gamma$-secretase complex that is essential for the generation of NICD (51), or anti-JAG1 antibody repressed TEA domain family member-dependent (TEADdependent) YAP/TAZ reporter activity in DKO primary hepatocytes as well as in YAP- or TAZ-expressing Huh7 cells (Figure 1G and Supplemental Figure 2, A and B). These results indicate that there is a positive feedback loop between Notch signaling and YAP/TAZ. To test whether Notch signaling activation enhances YAP/TAZ activity, we expressed NICD, and it indeed enhanced TEAD-dependent YAP/TAZ reporter activity (Figure 1H). To explore the mechanisms whereby NICD promotes YAP/TAZ activity, we first examined YAP and TAZ expression in various cell lines including Huh7 cells. We found that TAZ protein lev- els, and only slight amounts of YAP protein levels in different cell lines, were enhanced by NICDs (NICD1-4), while Taz mRNA expression levels were not altered (Figure 1I and Supplemental Figure 2, C-E). Furthermore, we examined TAZ protein degradation after treating the cells with the protein synthesis inhibitor cycloheximide (CHX) and found that NICD expression resulted in much-reduced TAZ protein degradation (Figure 1J). These results indicate that Notch signaling enhances YAP/TAZ activity by stabilizing TAZ protein through NICD.

To test whether NICD regulates TAZ protein stability by regulating Hippo signal transduction, we examined LATS activity and YAP phosphorylation in Huh7 cells and found that NICD had no influence, indicating that Notch signaling stabilizes TAZ protein without altering Hippo signaling (Supplemental Figure 2F). Therefore, we proceeded to ask whether NICD inhibits TAZ deg- 
radation directly by examining TAZ polyubiquitination. We found that NICD interacted with TAZ and reduced its polyubiquitination (Figure 1K and Supplemental Figure 3, A and B). Additionally, we determined that the $\mathrm{C}$-terminal region of TAZ was required for interaction with NICD (Supplemental Figure 3C), raising the possibility that association of NICD with TAZ may prevent the C-terminally phosphorylated TAZ from binding to $\beta$-TrCP, which promotes TAZ degradation (52). $\beta$-TrCP recognizes 2 different sites on the N-terminal (serine 58) or C-terminal (serine 314) region of TAZ when they are phosphorylated by glycogen synthase kinase 3 (GSK3) or casein kinase 1/LATS (CK1/LATS), respectively. Indeed, NICD disrupted the interaction between TAZ and $\beta$-TrCP (Figure 1L and Supplemental Figure 3D). Furthermore, we found that the N-terminal phospho-mutant TAZ-S58/62A could still be stabilized by NICD to a degree similar to that seen with WT TAZ, whereas the stabilized C-terminal phospho-mutants TAZ-S314A and TAZ-3SA, due to failure of $\beta$-TrCP binding, could not be further stabilized by NICD (Supplemental Figure 3E). Taken together, our results indicate that Notch signaling stabilizes TAZ protein by inhibiting its $\beta$-TrCP-mediated degradation, and by doing so, Notch signaling forms a positive feedback loop with YAP/TAZ.

Genetic removal of 1 copy of YAP is sufficient to completely restore the Hippo-mutant phenotype in the liver. Our results indicate that TAZ also plays important roles in the liver tumor formation and progression caused by Hippo signaling deficiency. To address the contribution of TAZ to liver enlargement and tumor formation, we generated Alb-Cre Mst1-/- Mst $2^{f l / f l} \mathrm{Taz}^{f l /+}$ (referred to hereafter as DKO-Taz $z^{f /+}$ ) mice by removing 1 allele of the Taz gene in the DKO liver. As with DKO-Yap $p^{f /+}$ mice, removal of 1 copy of Taz efficiently reduced liver overgrowth and tumor formation (Supplemental Figure 4). These findings show that TAZ is also required for the hepatomegaly and tumor initiation caused by Hippo deficiency.

Pharmacological or genetic blockade of Notch signaling suppresses YAP/TAZ activity in vivo. As YAP/TAZ promotes Jag1 expression and NICD stabilizes TAZ protein, our studies identify a potent positive feedback loop between YAP/TAZ and Notch signaling in the liver that may account for the rapid initiation and progression of liver tumors in Hippo-mutant mice. To test this hypothesis, we first inhibited Notch signaling pharmacologically in vivo by injecting DAPT into DKO mice. Breaking down the positive feedback loop by inhibiting the Notch pathways in DKO mice should reduce liver size and slow down tumor formation and progression. Indeed, DAPT injection led to reduced protein levels of JAG1, NICD, YAP, and TAZ (Figure 2A). Expression levels of YAP/TAZ and Notch target genes were also reduced in DAPT-treated mouse livers compared with expression levels in DMSO-injected controls (Figure 2, A and B). Consistent with these results, expression of oval/ductal cell markers was also significantly reduced (Figure 2, A and B). Importantly, DAPT injection resulted in a significant reduction of liver size, tumor numbers, and growth compared with DMSO-injected DKO control mice (Figure 2, C-F).

The Hippo-mutant liver exhibited increased Jag1 expression compared with other Notch ligands, and neutralization with anti-JAG1 antibody in vitro or DAPT injection in vivo efficiently inhibited the YAP/TAZ/Notch signaling feedback loop (Figures 1 and 2, and Supplemental Figure 1). To determine definitively whether Jag1 expression is critically required for liver tumor initiation and progression in DKO mice, we removed the Jag1 gene by generating $\mathrm{Alb}$-Cre $\mathrm{Mst1}^{-/-} \mathrm{Mst} 2^{\mathrm{f} / \mathrm{J}} \mathrm{Jag} \mathrm{1}^{\mathrm{fl/fl}}$ mice (referred to hereafter as TKO-Jag1 mice), which allowed for further investigation of the genetic interaction of Notch signaling with YAP/TAZ in liver size control and tumorigenesis. Consistent with the effects of DAPT injection, liver size, tumor numbers, and tumor burden were significantly reduced in the TKO-Jag1 mice compared with the control DKO mice (Figure 3, A-D). At the molecular level, the TKO-Jag1 mice showed reduced NICD and Notch signaling target gene expression as well as reduced YAP/TAZ protein levels compared with that observed in the DKO controls (Figure 3, $\mathrm{E}$ and $\mathrm{F}$ ). In addition, expression of the oval/ductal cell markers EPCAM and Sox9 was dramatically diminished in the TKO-Jag1 mouse liver (Figure 3, E-G). Taken together, these results indicate that upregulation of Jag1 is a critical downstream event leading to a positive feedback loop between the Notch signaling and YAP/ TAZ activity that promotes rapid tumor initiation and progression in the Hippo-deficient liver.

Wnt/ $\beta$-catenin signaling inhibits liver growth and tumor initiation in the Hippo-deficient liver. We have shown previously that Wnt/ $\beta$-catenin signaling might be activated in the DKO-mutant liver (25). To further test this, we examined the final outputs of Wnt/ $\beta$-catenin pathway and found that total and active $\beta$-catenin $(A B C)$ were markedly increased in the DKO liver and primary hepatocytes (Supplemental Figure 5, A-F). In addition, Wnt $/ \beta$-catenin reporter activity (Top-Flash) and expression of Wnt/ $\beta$-catenin target genes such as Axin2, Apcdd1, and Wif1 were also elevated in DKO hepatocytes (Supplemental Figure 5, G and $\mathrm{H})$. These results indicate that loss of the mouse Hippo kinases MST1 and MST2 indeed leads to Wnt/ $\beta$-catenin signaling activation in hepatocytes.

Because $\beta$-catenin has been found to cooperate with YAP/ TAZ to control heart size and colon cancer formation $(53,54)$, and activation of $\beta$-catenin leads to HCC (27), we expected that removal of $\beta$-catenin in the DKO liver might alleviate its phenotypes including liver enlargement and tumorigenesis. To test this idea, we crossed DKO mice with mice with a $\beta$-catenin-conditional null

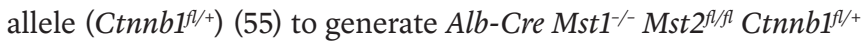
mice (referred to hereafter as DKO- $\beta c a t$-het mice) and Alb-Cre $M s t 1^{-/-} M s t 2^{f / / f l} C t n n b 1^{f / / f l}$ mice (referred to hereafter as TKO- $\beta c a t$ mice). Surprisingly, liver sizes in both the DKO- $\beta$ cat-het and TKO$\beta c a t$ mice were significantly enlarged compared with liver sizes in the DKO mice (Figure 4, A and B, and Supplemental Figure 6A). Consistent with these gross tissue abnormalities, hepatocyte proliferation indicated by phospho-histone 3 staining was upregulated to a greater degree in TKO- $\beta$ cat livers than in DKO livers, whereas cell death detected by TUNEL assay was reduced (Figure 4, C and D, and Supplemental Figure 6, B and C). Strikingly, liver tumorigenesis in the DKO mice was further accelerated by $\beta$-catenin removal. In a cohort of control and mutant mice, we observed that by 2 months of age, $100 \%$ and $42 \%$ of TKO- $\beta$ cat and DKO- $\beta$ cathet mice, respectively, had already developed HCC, while none of the DKO mice had developed tumors. At 3 months of age, approximately $40 \%$ of the DKO mice developed HCC, whereas almost all TKO- $\beta c$ at and DKO- $\beta$ cat-het mice had HCC, with markedly increased numbers of tumor nodules (Figure 4E and Supplemental 
A
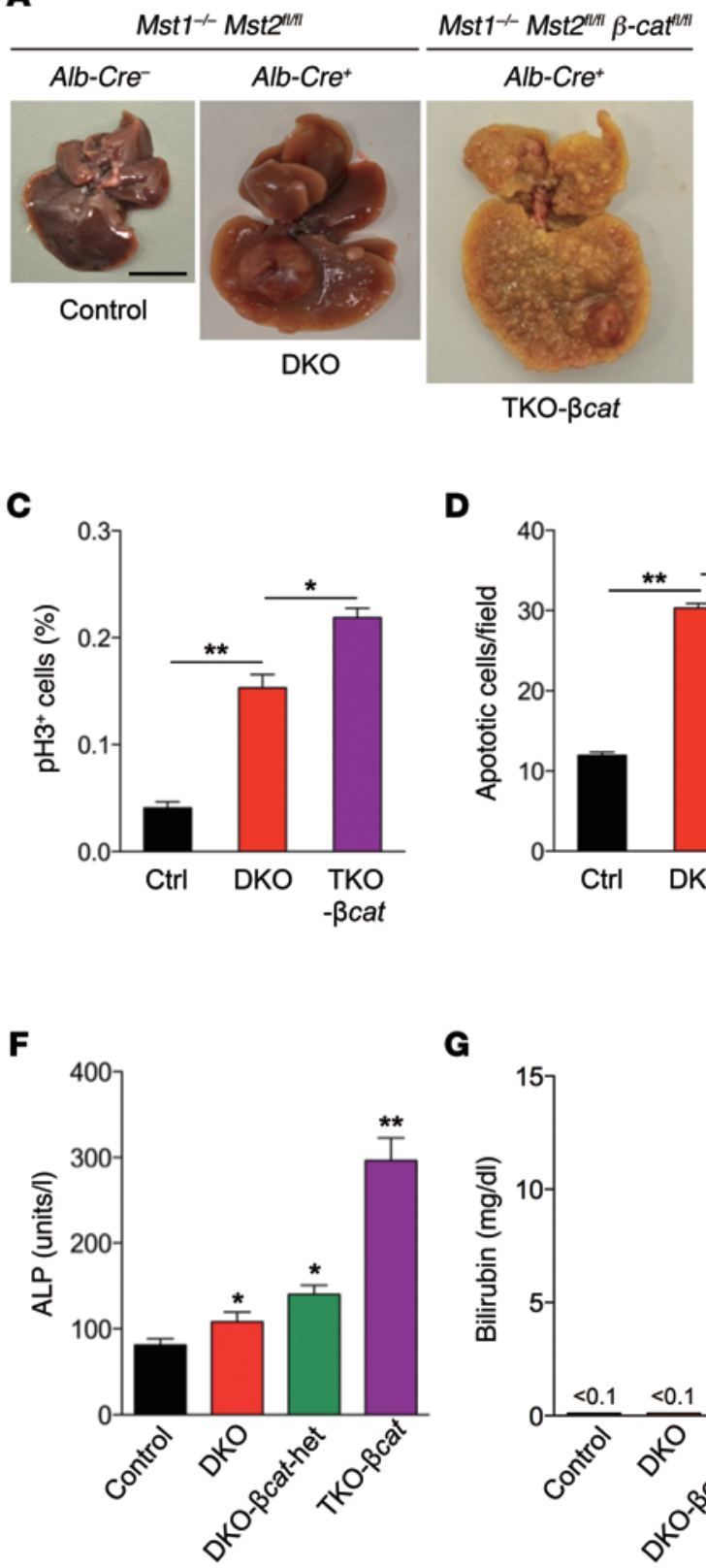

I

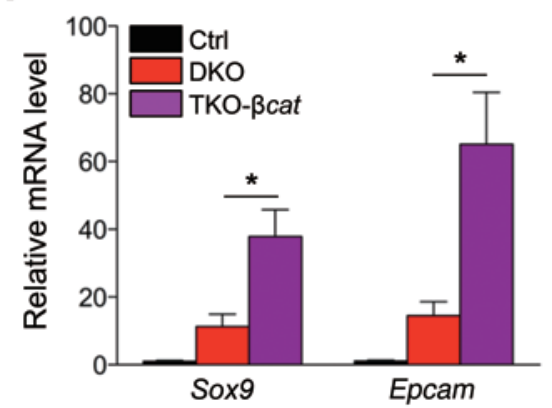

D
B
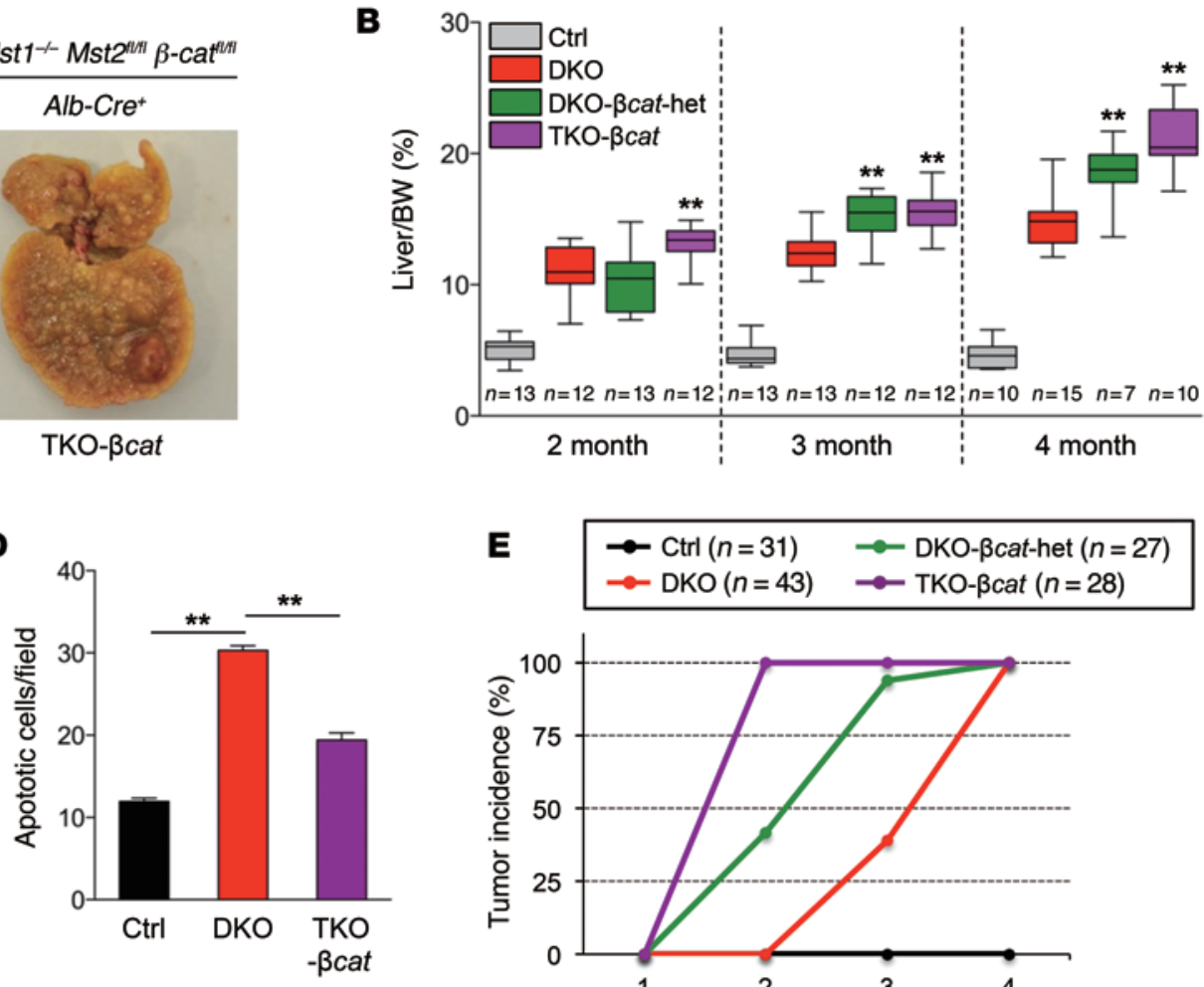

E $\quad \begin{array}{ll}\rightarrow \text { Ctrl }(n=31) & \rightarrow \text { DKO- } \beta \text { cat-het }(n=27) \\ \rightarrow \text { DKO }(n=43) & \rightarrow \text { TKO- } \beta \text { cat }(n=28)\end{array}$

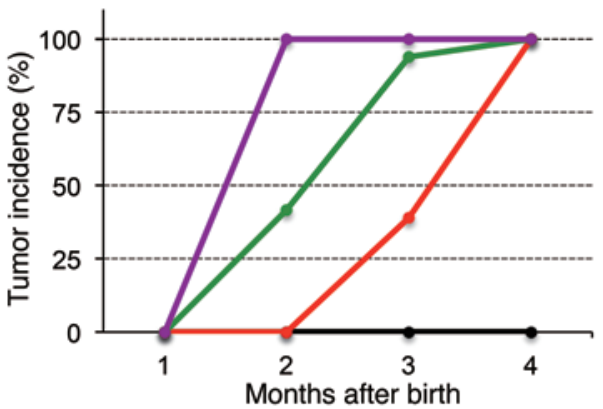

H

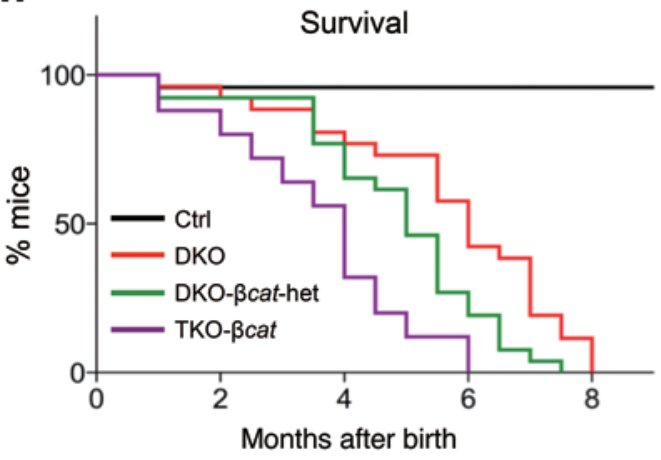

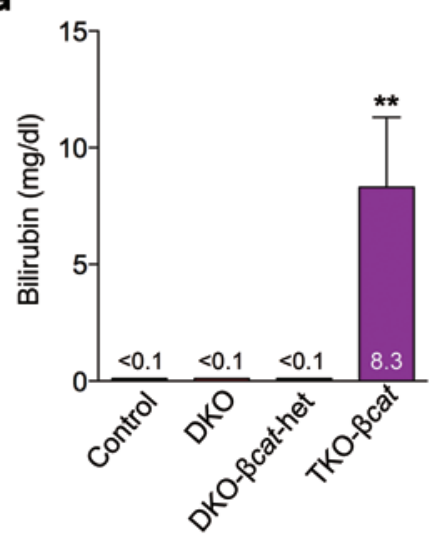

J

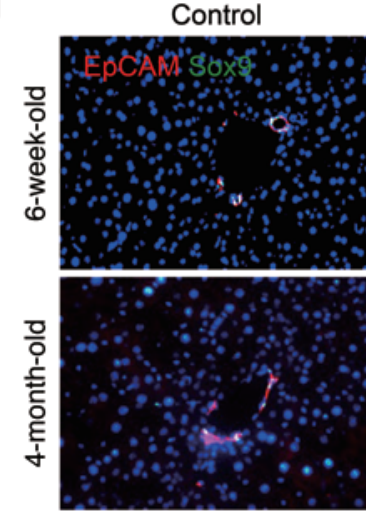

DKO

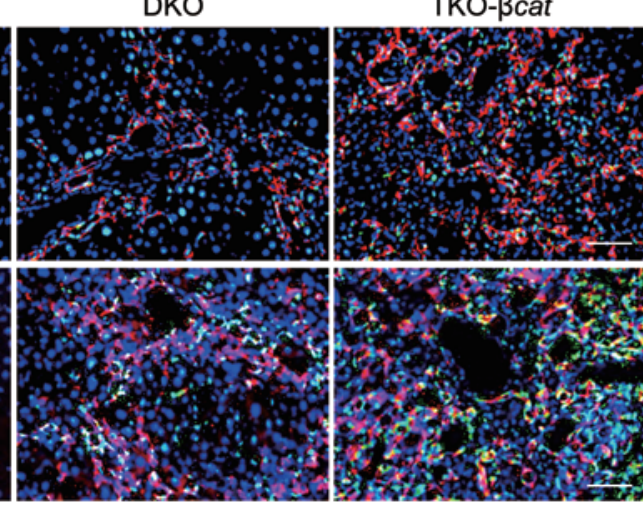


Figure 4. Loss of Wnt/ $\beta$-catenin signaling enhances DKO liver phenotypes. (A) Representative macroscopic images of livers from 4-month-old mice of the indicated genotypes. Scale bars: $1 \mathrm{~cm}$. (B) Analysis of liverto-BW ratio of mice of the indicated genotypes and ages. Middle bars in the box plots represent the median value $(n=7-15)$. (C) Quantification of proliferative cells in the indicated mouse liver tissues ( $n=4$ mice per genotype). (D) Quantification of TUNEL ${ }^{+}$cells. The number of TUNEL+cells/ $\mathrm{mm}^{2}$ was counted for each sample in a group ( $n=4$ mice per genotype). (E) Quantification of liver tumor incidence according to the indicated genotypes and ages. ( $\mathbf{F}$ and $\mathbf{G}$ ) Serum levels of ALP and bilirubin in mice of the indicated genotypes. (H) Kaplan-Meier curves showing survival of mice of the indicated genotypes $(n=24-26) . P=0.0104$ for DKO versus DKO- $\beta$ cathet; $P=0.0002$, for DKO versus TKO- $\beta$ cat. (I and J) qRT-PCR analysis ( $n=3$ per group) (I) and immunofluorescence staining (J) for the oval cell markers Sox9 and EPCAM in liver samples from 6-week-old and 4-month-old mice of the indicated genotypes. Scale bars: $100 \mu \mathrm{m}$. Data are expressed as the mean \pm SEM. ${ }^{*} P<0.05$ and ${ }^{* *} P<0.01$, by 1 -way ANOVA with Tukey's posthoc test (B-D, F, G, and I) when ANOVA was significant.

Figure 7A). In some liver tumor samples, we also found intrahepatic cholangiocarcinoma (ICC), composed mainly of multilayered biliary epithelial cells (Supplemental Figure 7B). In the MST1/2, $\beta$-catenin TKO mouse line, we found a larger number of less-differentiated HCCs and ICCs, but substantially fewer hepatocellular ademomas (HCAs) compared with our findings in the MST1/2 DKO mouse line. These results demonstrate genetically that $\beta$-catenin inhibits the liver tumor initiation caused by Hippo signaling inactivation. Apart from hepatomegaly and HCC formation, the TKO- $\beta c a t$ and DKO- $\beta c a t$-het mice also showed other more severe liver phenotypes compared with the DKO mice, including a distended abdomen and lethargy at earlier ages. In particular, livers and serum from TKO- $\beta$ cat mice were abnormally yellow due to severe jaundice, as evidenced by elevated serum levels of alkaline phosphatase (ALP) and bilirubin (Figure 4, A, F and G, and Supplemental Figure $7 C$ ). The combination of these effects resulted in an earlier death of the DKO- $\beta$ cat-het and TKO- $\beta$ cat mice compared with the DKO mice, with death of the TKO- $\beta$ cat mice occurring at the earliest age (Figure $4 \mathrm{H})$. It is important to note that $\beta$-catenin, in addition to being a regulator of Wnt signaling, is also associated with E-cadherin at the cell membranes. The function of $\beta$-catenin in Wnt signaling is nonredundant, but previous reports suggest that its function in cell adhesion by binding to E-cadherin can be compensated by $\gamma$-catenin in hepatocytes $(56,57)$. We indeed observed a robust increase in $\gamma$-catenin in the livers of TKO- $\beta$ cat (Supplemental Figure 8, A and B). $\gamma$-Catenin had also functionally replaced $\beta$-catenin in cell adhesion, as E-cadherin mainly interacted with $\beta$-catenin in the DKO livers, while it was associated with $\gamma$-catenin in TKO- $\beta$ cat livers (Supplemental Figure $8 C$ ). However, $\gamma$-catenin could not compensate for $\beta$-catenin's function in Wnt signaling, as it was unable to activate TCF reporter activity (Supplemental Figure 8D). These data indicate that complete loss of $\beta$-catenin did not disrupt cell adhesion at the hepatocyte membrane in TKO- $\beta$ cat mice due to the compensatory upregulation of $\gamma$-catenin. To further test the transcriptional role of $\beta$-catenin in inhibiting rapid HCC formation and hepatomegaly, we injected iCRT3, a small-molecule inhibitor of Wnt signaling, into DKO mice. iCRT3 is known to disrupt $\beta$-catenin interaction with TCF factors, without altering its protein levels (58). Injection of iCRT3 into DKO mice led to a marked reduction in Wnt target genes including APC downregu- lated 1 (Apcdd1) and Wnt inhibitory factor 1 (Wifl) (Supplemental Figure 8E). However, iCRT3 injection significantly enhanced YAP/ TAZ target gene expression and the DKO liver phenotype (Supplemental Figure 8, F and G). Taken together, these data indicate that Wnt/ $\beta$-catenin signaling suppresses liver enlargement and tumor initiation in the Hippo-mutant liver.

Wnt/ $\beta$-catenin signaling inhibits YAP/TAZ/Notch positive feedback activation by regulating DP1 localization. The unexpected suppressive function of $\beta$-catenin in liver size control and tumor initiation in the absence of MST1 and MST2 prompted us to investigate the underlying molecular mechanisms. We found that EPCAM and Sox9 expression and the oval/ductal cell population was more severely expanded in the TKO- $\beta$ cat liver compared with the DKO liver (Figure 4, I and J), suggesting that activated $\beta$-catenin in the DKO liver may repress YAP/ TAZ activity by inhibiting Notch signaling. Indeed, we found that Notch signaling was more activated in the TKO- $\beta$ cat liver than in the DKO liver, as determined by expression of Jag1, NICD, and Notch signaling target genes (Figure 5, A and B). TAZ protein levels were higher in TKO- $\beta$ cat liver than in DKO liver, and this was consistent with higher Notch signaling in TKO- $\beta$ cat liver (Figure 5A). Notch signaling was more active in tumor nodules than in the surrounding nontumor tissue. The tumor nodules also contained more oval/ductal cells than did the neighboring nontumor regions, as shown by JAG1 and Sox9 expression levels (Supplemental Figure 9A). Furthermore, injection of DAPT into the TKO- $\beta$ cat mice led to a marked reduction in protein levels of JAG1 and NICD as well as YAP and TAZ. Also, it significantly reduced the expression of Notch target genes Nrarp and Hes 1 (Figure 5, B and C). Consistent with these molecular changes, DAPT-treated TKO- $\beta$ cat mice had a significant reduction in liver size, tumor numbers, and other liver malfunctions indicated by serum levels of ALP and bilirubin (Figure 5, D-G). These results suggest that the Wnt/ $\beta$-catenin pathway may inhibit Notch signaling in the liver. In support of this idea, we found that overexpression of $\beta$-catenin suppressed Notch reporter activity in Huh7 cells (Supplemental Figure 9B). In Alb-Cre Ctnnb1//Aex3 mice, which produce truncated, nondegradable $\beta$-catenin in hepatocytes, Notch signaling and oval/ductal cells were reduced (Supplemental Figure 9C), while removal of $\beta$-catenin from Alb-Cre Ctnnb1 ${ }^{f / f l}$ mouse hepatocytes showed the opposite effect (Supplemental Figure 9D).

To understand the mechanism whereby Wnt/ $\beta$-catenin signaling inhibits Notch signaling, we came across dimerization partner 1 (DP1), which was originally identified as a binding partner of the E2Fs transcriptional factor-regulating genes involved in $\mathrm{G}_{1} / \mathrm{S}$ transition and DNA synthesis $(59,60)$. Our recent work showed that DP1 plays dual roles in regulating the Wnt/ $\beta$-catenin pathway during anteroposterior neural patterning, and these dual functions are determined by Wnt activity-dependent differential localization of DP1 (61). In the Wnt-low region, DP1 is mainly localized in the cytoplasm. When Wnt signaling is high, DP1 enters the nucleus, where it boosts Wnt signaling (61). Interestingly, DP1 was predominantly cytoplasmic in the hepatocytes of control mice (Figure $5 \mathrm{H}$, left panel), but it was enriched in the nucleus of DKO hepatocytes in which Wnt/ $\beta$-catenin signaling was upregulated (Figure $5 \mathrm{H}$, middle panel). When $\beta$-catenin was removed in the TKO- $\beta$ cat 
A

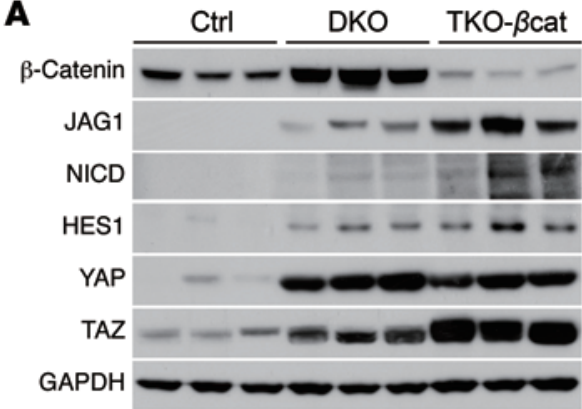

B

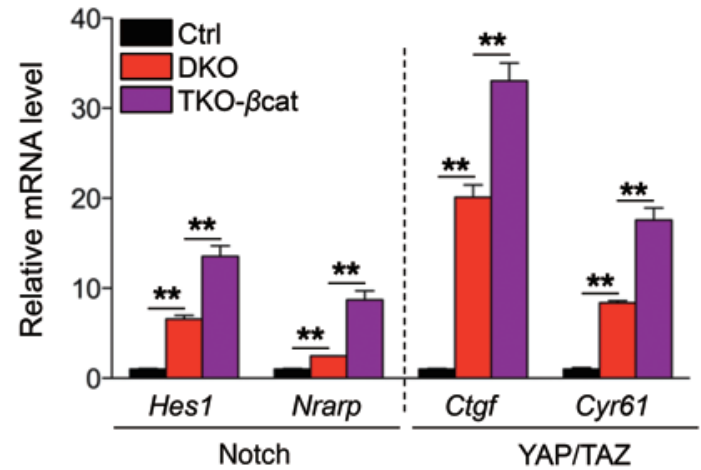

C

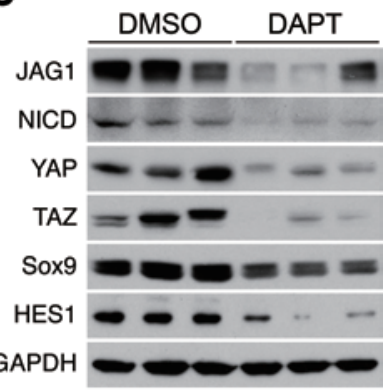

TKO- $\beta$ cat

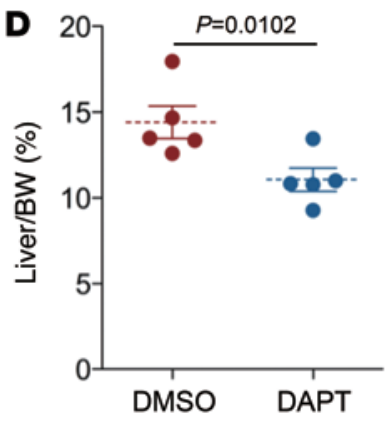

H Control
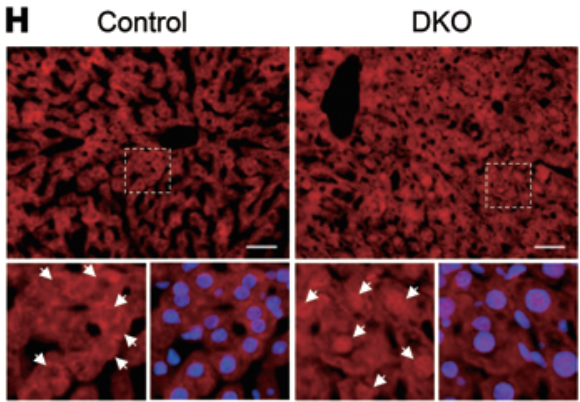

E

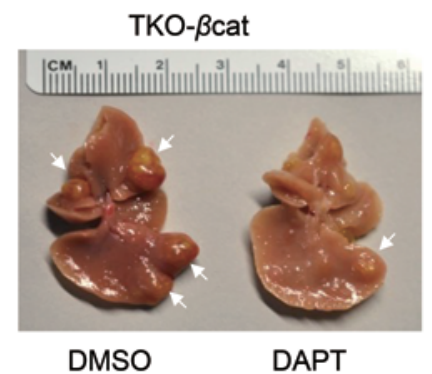

TKO-ßcat

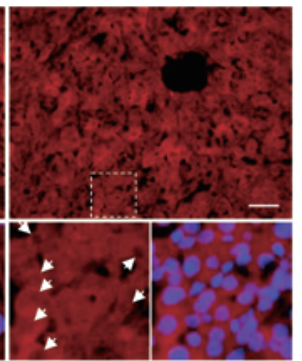

DP1/DAP

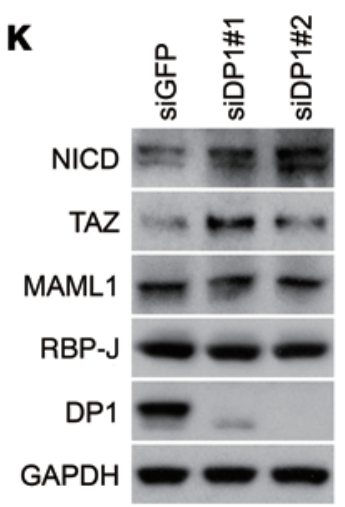

$\mathbf{L}$

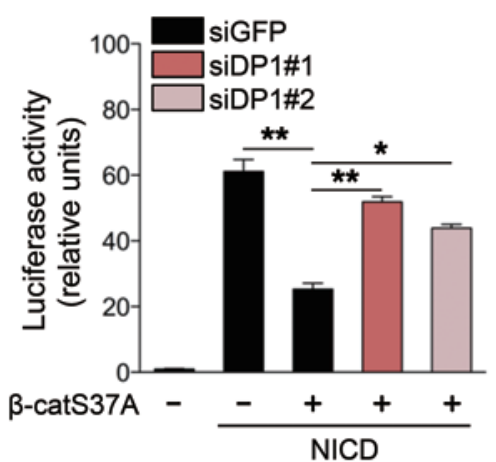

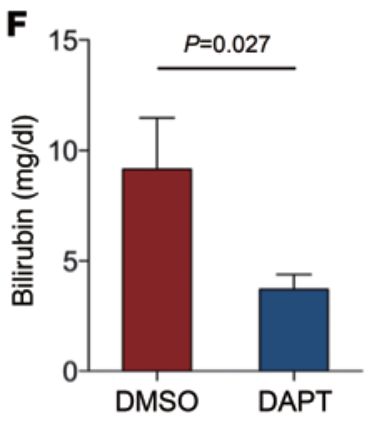

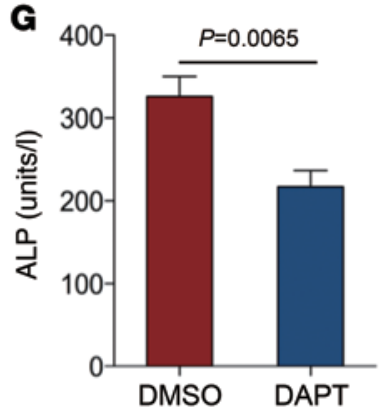

I

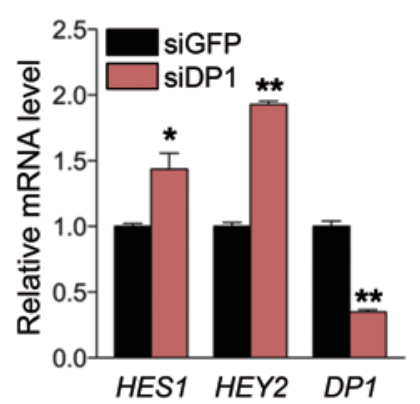

J

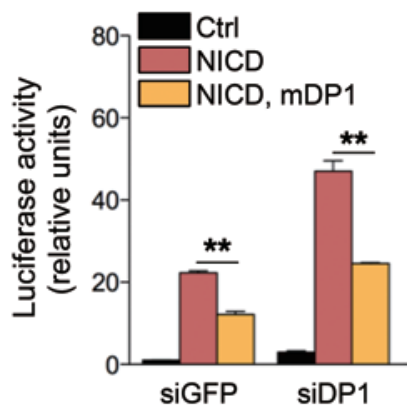

Figure 5. Wnt/ $\beta$-catenin signaling inhibits YAP/TAZ/Notch positive feedback activation by promoting DP1 nuclear localization. (A) Western blot analysis of control, DKO, and TKO- $\beta$ cat liver tissue for the indicated proteins. (B) qRT-PCR analysis of Notch and YAP/TAZ target gene expression in liver from mice of the indicated genotypes ( $n=3$ per group). (C) TKO- $\beta$ cat mice were given 20 i.p. injections of DAPT at a concentration of $125 \mathrm{mg} / \mathrm{kg}$ every other day. Western blot analysis of liver lysates from DAPT- or DMSO-injected TKO- $\beta$ cat mice. (D-G) Analysis of liver-to-BW ratio (D), liver tumor size (E), serum levels of bilirubin (F) and ALP (G) in DMSO- or DAPT-injected TKO- $\beta$ cat mice ( $n=5$ per group). (H) DP1 staining was performed on liver sections from 6 -week-old mice of the indicated genotypes. Scale bars: $50 \mu \mathrm{m}$. Original magnification: $\times 25$. (I) qRT-PCR analysis of Notch target gene expression in DP1-depleted cells $(n=3)$. (J) Notchdependent reporter assay in Huh7 cell-depleted human DP1. The effect of DP1 loss on Notch reporter activity was rescued by overexpression of siRNA-resistant murine DP1 (mDP1) $(n=3)$. (K) Western blot analysis with the indicated antibodies in DP1-depleted Huh7 cells. (L) Notch reporter assay in Huh7 cells transfected with the indicated plasmids and $\operatorname{siDP1}(n=3)$. (M) Suppression of NICD-mediated reporter activity by WT DP1 or NLS-DP1, but not by NES-DP1 $(n=3)$. Data are expressed as the mean \pm SEM. ${ }^{*} P<0.05$ and ${ }^{* *} P<0.01$, by 2-tailed Student's $t$ test $(\mathbf{F}, \mathbf{G}$, and $\mathbf{I})$ and 1 -way ANOVA with Tukey's post-hoc test (B, J, L, and $\mathbf{M}$ ) when ANOVA was significant. 
hepatocytes, DP1 nuclear localization was reduced (Figure 5H, right panel). This finding demonstrates that localization of DP1 in hepatocytes depends on Wnt/ $\beta$-catenin signaling activity and that $\beta$-catenin is required to promote DP1 nuclear localization. To test whether nuclear localization of DP1 induced by Wnt signaling also modulates other signaling activities, we first examined whether DP1 is able to regulate the Notch signaling pathway. Overexpression of DP1 significantly repressed Notch reporter activity in Huh7 or HeLa cells (Supplemental Figure 9, E and F). Conversely, knocking down DP1 by siRNA further enhanced Notch reporter activity as well as expression of the target genes HES1 and HEY2 in Huh7 cells (Figure 5I and Supplemental Figure 9, G and H), and these effects could be rescued by expressing mouse $D p 1$ that could not be targeted by the siRNA recognizing the human DP1 gene (Figure 5J). Furthermore, DP1 also acted in vivo to inhibit Notch signaling, since depletion of DP1 by a morpholino (MO) targeting DP1 enhanced expression of the Notch target gene her6 in zebrafish embryo, as analyzed by ISH (Supplemental Figure 9I).

To understand the molecular mechanism by which DP1 suppresses the Notch pathway, we tested the levels of core transcription components in Notch signaling and found that overexpression or knockdown of DP1 reduced or increased NICD, respectively, without altering other core components such as mastermind-like 1 (MAML1) and RBP-J. In addition, reduction of NICD protein by DP1 was fully rescued by treatment with the proteasome inhibitor MG132, indicating that DP1 regulates ubiquitin-proteasome-mediated NICD degradation (Figure $5 \mathrm{~K}$ and Supplemental Figure 9J). NICD contains the PEST degradation domain, which can be recognized and subsequently degraded by the E3 ubiquitin ligase FBW7. NICD degradation by DP1 was abolished when its PEST domain (NICD $\triangle \mathrm{PEST}$ ) was deleted (Supplemental Figure 9K). Likewise, overexpression or depletion of DP1 no longer affected the Notch reporter activity induced by NICD $\triangle$ PEST (Supplemental Figure $9 \mathrm{~L}$ and M). Taken together, these results suggest that DP1 inhibits Notch signaling by promoting NICD degradation. We further asked whether inhibition of Notch signaling by $\beta$-catenin can be alleviated by knocking down DP1 expression in Huh7 cells and found that this is indeed the case, indicating that DP1 mediates at least part of the role of Wnt/ $\beta$-catenin in inhibiting Notch signaling (Figure $5 \mathrm{~L}$ ). We then asked more specifically whether nuclear localization is required for DP1 to inhibit Notch signaling and found that only nuclear DP1 (NLS-DP1), but not cytoplasmic DP1 (NES-DP1), was able to suppress Notch activity (Figure $5 \mathrm{M}$ ). Taken together, our data show that Wnt/ $\beta$-catenin signaling inhibits the YAP/TAZ/Notch positive feedback loop by controlling DP1 nuclear localization. Our results provide definitive evidence that HCCs formed primarily by Wnt/ $\beta$-catenin signaling activation or YAP/TAZ/ Notch signaling activation are fundamentally different in both their underlying cellular and molecular mechanisms, suggesting that different therapeutic strategies must be applied to treat these 2 types of HCCs.

STAT3 activation is not required for HCC formation caused by loss of MST1 and MST2. We have previously shown that there were signs of increased inflammation in the histological analysis of DKO liver $(25,39,62)$ (Figure 6A). Since inflammatory responses are known to be associated with a variety of human cancers, we first examined Kupffer cells that are macrophages in the liver in order to understand how inflammatory responses in the Hippo-deficient DKO liver contribute to liver tumor progression. Kupffer cells can regulate liver proliferation and survival by secreting cytokines $(63,64)$. We found that Kupffer cells, in particular the newly infiltrated Kupffer cells associated with inflammation, which are the $\mathrm{CD} 11 \mathrm{~b}^{+} \mathrm{F} 4 / 80^{+}$cell population, were markedly increased in DKO livers (Figure 6A and Supplemental Figure 10A). Consistent with these changes, we found that the proinflammatory IL-6 family of cytokines including IL-6, IL-11, leukemia-inhibitory factor (LIF), and oncostatin M (OSM), known to be released in newly infiltrating Kupffer cells, were highly expressed in DKO-mutant liver when we analyzed the IL-6/ STAT3 signaling pathway using PCR array (Figure 6B and Supplemental Figure 10B). The expression was further corroborated by quantitative real-time PCR (qRT-PCR) analysis (Figure 6C). As these cytokines signal through activating STAT3, which is required for tumorigenesis in mice $(4,65)$, we examined STAT3 activation and found that both STAT3 total protein levels, STAT3 phosphorylation, and its target genes $\mathrm{Bcl}-2$ and Socs3 were augmented in the DKO liver (Figure 6, D and E), indicating that STAT3 signaling was indeed activated in the DKO liver.

As STAT3 is associated with a wide variety of human malignancies and known to be activated in the majority of HCCs with poor prognosis and not in surrounding nontumor tissue or in normal liver (4), we hypothesized that STAT3 activation in the DKO liver may mediate the effects of loss of Hippo signaling in causing HCC. Similar signaling regulation between Hippo and Jak/STAT pathways has been found in Drosophila, in which loss of Hippo signaling promotes intestinal stem cell proliferation by activating the Jak/STAT pathway (66-68). To test the contribution of STAT3 activation to HCC formation in the DKO liver, we removed Stat3 in DKO liver by generating Alb-Cre $\mathrm{Mst1}^{-/-} \mathrm{Mst} 2^{f / f l}$ Stat $^{\text {fl/fl }}$ (TKO-Stat3) mice. However, to our surprise, tumor formation and progression were the same in both TKO-Stat 3 and DKO livers. We observed no significant reduction in the number of tumors or size of livers (Figure 6, F-H). Efficient STAT3 removal was documented by Western blot analysis, and consistent with a gross analysis of the liver, we observed no reduction of YAP or Sox9 protein levels (Figure 6I). Furthermore, Kupffer cell numbers and YAP target gene expression levels were not altered in the TKO-Stat3 liver compared with that observed in the DKO liver (Figure 6J and Supplemental Figure 11A). We also tested the expression of several inflammatory cytokines and STAT3 target genes. Interestingly, most inflammatory cytokines and chemokines tested were not significantly altered in TKO-Stat 3 livers when compared with those in DKO livers, even though STAT3 target genes such as Bcl-2 and Socs3 were reduced in the TKO-Stat3 liver (Supplemental Figure 11, A and B). These results suggest that loss of STAT3 in hepatocytes may not be sufficient to reduce the inflammatory response that occurred in the DKO mice. To test whether STAT3 inhibitors could still be effective therapeutically if the target cell population was macrophages or other nonparenchymal cell types, we injected Stattic, a small-molecule inhibitor of STAT3 (69), into DKO mice and found that the inhibitor reduced STAT3 target gene expression (Supplemental Figure 11C). However, STAT3 inhibition by Stattic 
A

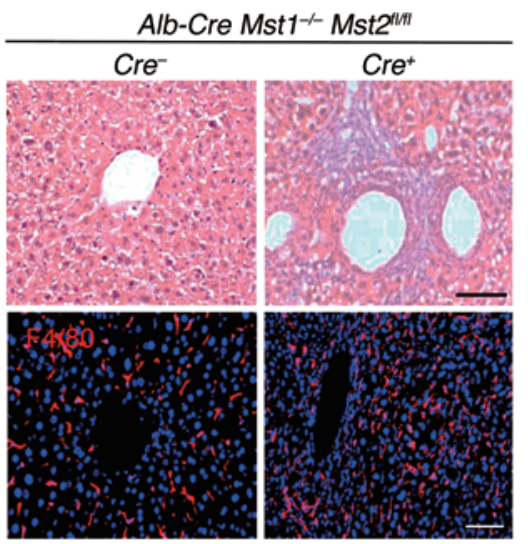

D

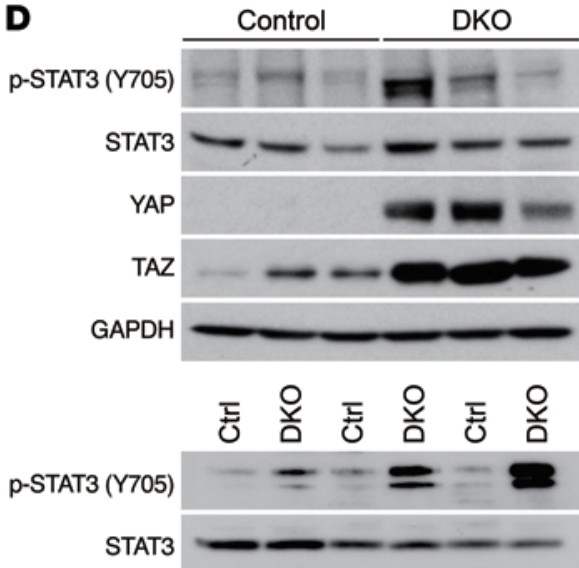

G

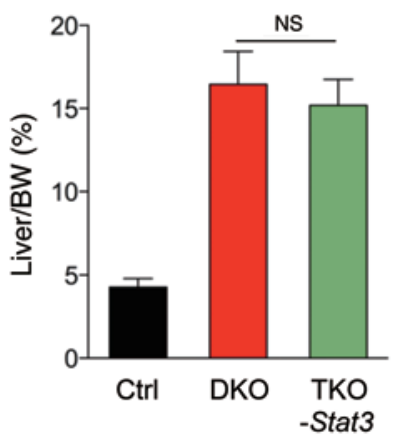

B

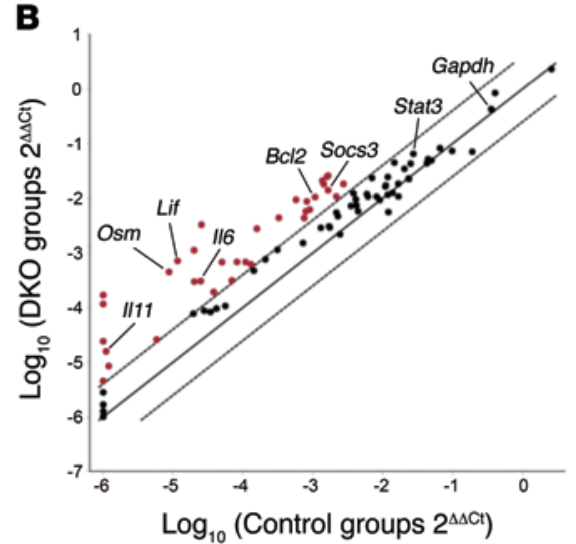

E

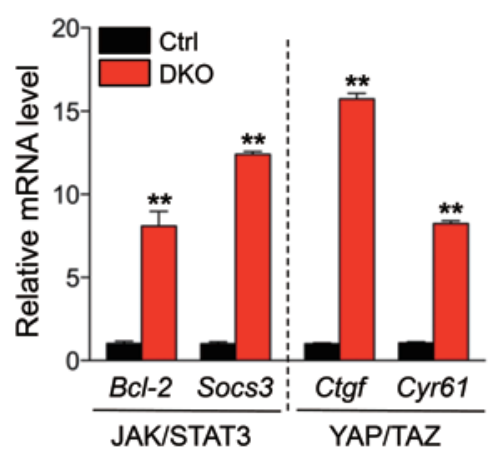

H

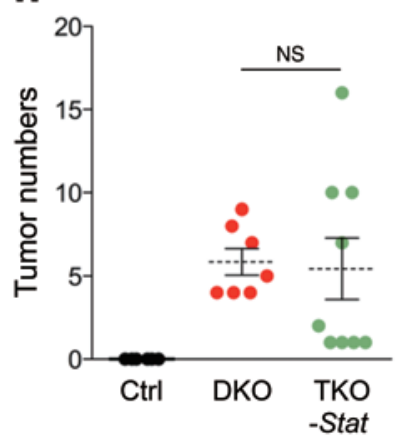

C

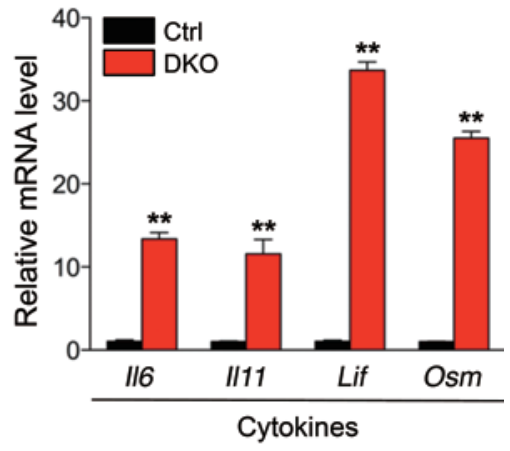

J

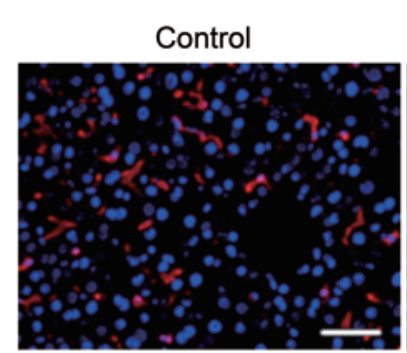

DKO

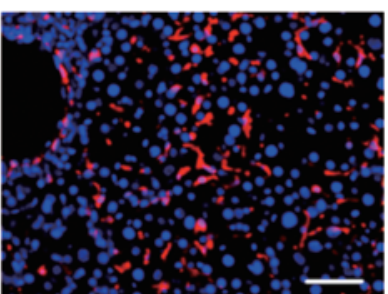

F4/80/DAPI

F Control

DKO
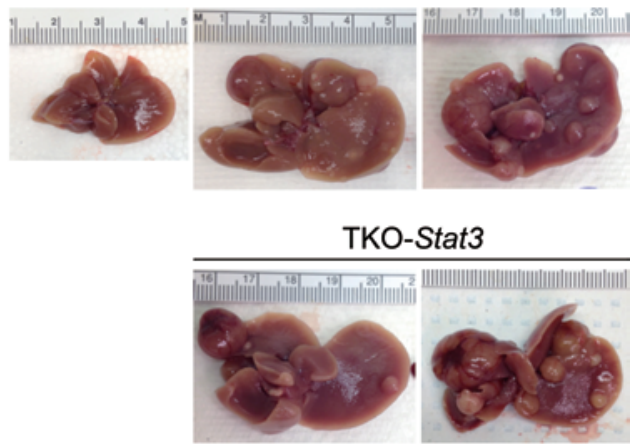

I
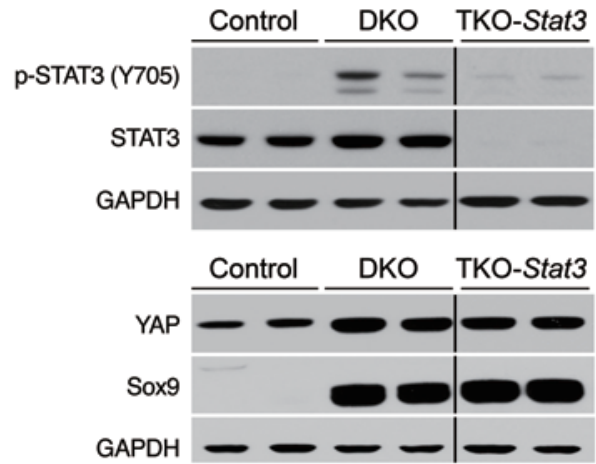

TKO-Stat3

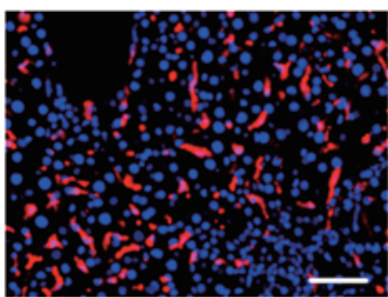

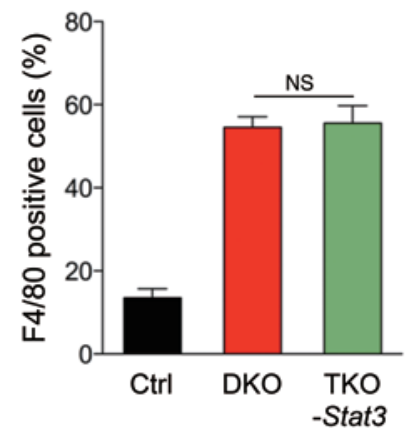


Figure 6. Stat3 ablation in liver failed to alleviate the liver phenotype of DKO mice. (A) Representative H\&E-stained liver sections from 2-monthold mice of the indicated genotypes (top). Scale bars: $50 \mu \mathrm{m}$. Liver sections from 2-month-old mice were subjected to immunofluorescence staining for anti-F4/80 antibody (bottom). (B) Scatter plot analysis for $84 \mathrm{IL}-6 /$ STAT3-related genes in control and DKO mouse liver. The figure depicts a log transformation plot of the relative expression levels of each gene $(\Delta \Delta \mathrm{Ct})$ in control and DKO groups. Dotted lines indicate a 4 -fold change in the gene expression threshold. (C) qRT-PCR of IL-6 cytokine family genes for control and DKO liver $(n=3)$. (D) Western blot analysis of liver extracts from control and DKO mice at 4 months of age using the indicated antibodies. STAT3 phosphorylation (Y705) levels were normalized to total STAT3 protein (bottom panel). p-STAT3, phosphorylated STAT3. (E) qRT-PCR of the STAT3 target genes Bcl-2 and Socs3 with the indicated liver samples $(n=3)$. (F-H) Representative macroscopic images of liver (F), analysis of liver-to-BW ratio (G), and average number of liver tumors (H) in 4-month-old mice of the indicated genotypes. (I) Western blot analysis of 4-month-old TKO-Stat3 mouse liver using the indicated antibodies. (J) Liver sections were subjected to immunofluorescence staining with $F 4 / 80$ antibody, and the percentage of $F 4 / 80^{+}$cells was quantified. Scale bars: $100 \mu \mathrm{m}$. Data are expressed as the mean \pm SEM. ${ }^{*} P<0.01$, by 2 -tailed Student's $t$ test (C and $\mathbf{E}$ ) and 1-way ANOVA with Tukey's post-hoc test ( $\mathbf{G}, \mathbf{H}$, and $\mathbf{J})$.

did not induce a significant alteration in the DKO liver phenotype (Supplemental Figure 11, D and E). Therefore, despite the upregulated proinflammatory response in the DKO liver, our data indicate that STAT3 activation is not required for the tumor formation caused by loss of Mst1 and Mst2.

\section{Discussion}

In this study, we have identified an interacting network of Hippo/Wnt/ $\beta$-catenin/Notch signaling that governs liver growth and represses tumor initiation through rigorous in vivo genetic studies. This in vivo network approach allowed us to identify both positive and surprising suppressive interactions of protumor formation pathways that exist in the same tumor, highlighting the necessity to develop context-dependent therapeutic approaches to treating tumors.

It has been well established that HCC formation is critically controlled by both genetic and environmental factors. Indeed, in many HCC genetic models, HCC formation was detected either very late in the life of the mouse, or was caused by a genetic lesion(s) and another environmental insult such as injection of DEN $(1,6)$. In this regard, it is remarkable and unique that HCC formed quickly in early life of the MST1/2 DKO mice (readily detected at 3 months after birth), without any environmental insult. Therefore, it is likely that loss of MST1/2 and/or the resultant upregulation of $\mathrm{YAP} / \mathrm{TAZ}$ activity is centrally important in promoting tumor initiation by controlling hepatocyte and oval cell proliferation. Other genetic and environmental factors may regulate hepatocytes and/or oval cell proliferation by interacting with the Hippo/YAP/TAZ pathway. Here, we show that a strong positive feedback loop between YAP/TAZ and Notch signaling contributes significantly to both liver cell fate determination and rapid HCC formation in Mst1/2 DKO mice. This vicious positive feedback loop suggests that even subtle alterations in Hippo signaling in human cancer can be amplified and result in substantial changes in hepatocyte proliferation and differentiation due to the interaction of Hippo with other signaling pathways.
YAP/TAZ and Notch signaling activities are higher in biliary duct epithelial cells, which are located around the portal vein and give rise to oval cells $(38,44)$. The positive feedback loop is essential to maintaining the high Notch signaling activity that is required for ductal and oval cell fate determination and maintenance, as loss of YAP1 results in much-reduced expression of Sox9, a marker of ductal and oval cell fates (70). MST1 and MST2 are required to keep the positive feedback loop in check in normal differentiated hepatocytes by inhibiting the activities of YAP/ TAZ and Notch signaling. It will be very interesting to identify the mechanism responsible for the difference in YAP/TAZ activity between oval/ductal cells and hepatocytes. In this regard, MST1 and MST2 act as critical gatekeepers in normal proliferation-competent cells by regulating 2 proproliferation signaling pathways at the same time. Loss of MST1 and MST2 only in Sox $9^{+}$cells led to biliary duct hyperplasia, without HCC formation (Supplemental Figure 12), suggesting that the oval cells formed by hepatocyte reprogramming are a critical cellular mechanism underlying the rapid tumor initiation and progression in Mst1/2 DKO livers.

Surprisingly, we found that Wnt/ $\beta$-catenin signaling inhibited YAP/TAZ-dependent liver growth and HCC formation. Consistent with recent findings, we did not observe direct regulation of YAP or TAZ protein levels or activity by the Wnts or $\beta$-catenin $(71,72)$. The negative regulation of the YAP/TAZ/Notch positive feedback loop by Wnt/ $\beta$-catenin signaling is critically important in both cell fate regulation, metabolic zonation (73), and tumor formation in the liver (Figure 7). Unlike the Notch signaling that is higher around the portal vein area and promotes biliary ductal cell differentiation, Wnt/ $\beta$-catenin signaling forms a high-to-low gradient from the central vein to the portal vein and favors hepatocyte fate while inhibiting ductal and oval cell fate choices (74). Loss of $\beta$-catenin in DKO mice further enhanced hepatocyte reprogramming into oval cells as well as cell proliferation, leading to a more severe expansion of the oval cell population, increased tumor nodule numbers, and earlier tumor initiation. Likewise, earlier work in chronic liver injury models showed that divergent specification of liver progenitors is directed by alternatively activating Wnt or Notch signaling during liver regeneration (74). Numb was shown to act as a "switcher" for Wnt-mediated Notch inhibition in liver regeneration. Here, we showed that nuclear localization of DP1 by activation of Wnt/ $\beta$-catenin signaling is also required to repress Notch signaling during liver tumorigenesis. Therefore, besides the developmental role of DP1 described in previous work (61), our findings indicate that Wnt-dependent DP1 nuclear localization may be involved in liver tumor formation and that DP1 can function as a mediator in Wnt signaling-dependent Notch regulation.

It is likely that the functional output of YAP- $\beta$-catenin interaction has tissue-dependent differential functions in Hippo-mutant mice. Findings similar to ours have been reported with regard to the role of $\beta$-catenin as an inhibitor of TAZ in liver cancer cells or APC-deficient mouse livers (75). Furthermore, while conditional knockout of Salvador in developing hearts displayed cardiomegaly, together with elevated Wnt/ $\beta$-catenin signaling, heterozygous deletion of $\beta$-catenin was found to relieve cardiomyocyte proliferation and heart size, indicating that the Wnt/ $\beta$-catenin pathway is required for YAP activity to control cardiac organ size (53). Likewise, YAP is essential for $\beta$-catenin nuclear accumulation in intestinal 


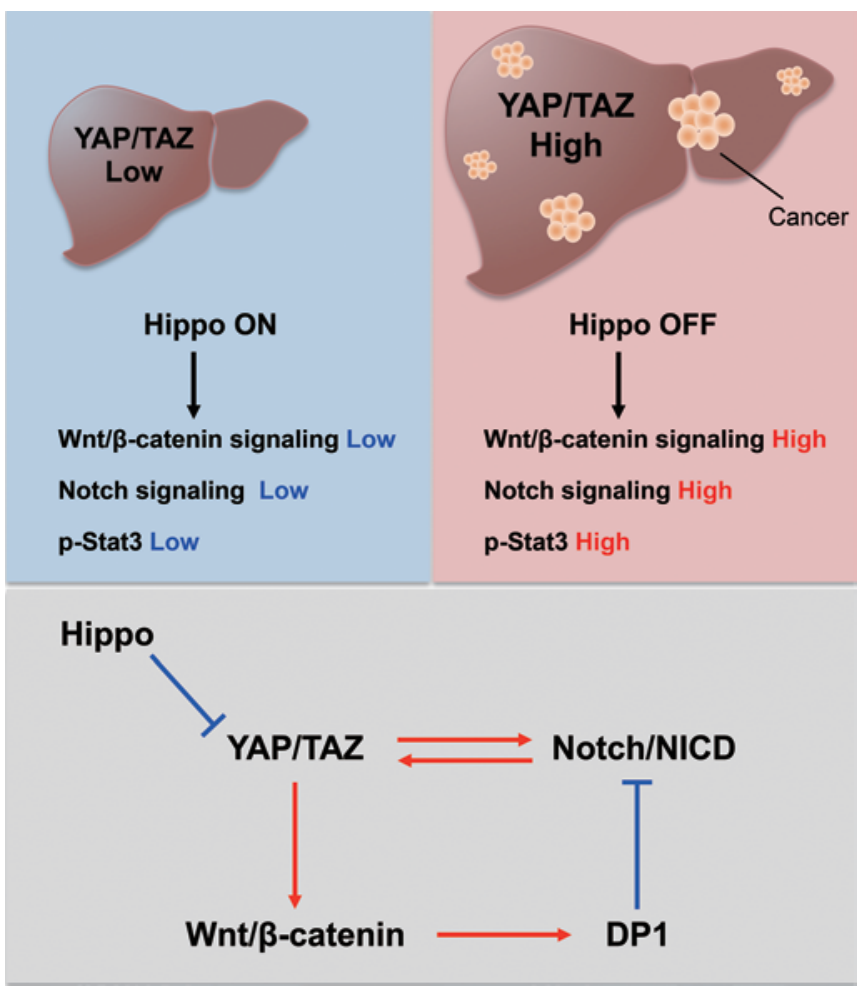

Figure 7. Proposed model of an interacting network of Wnt/ $\beta$-catenin, Notch, and Hippo signaling in the regulation of liver tumor formation. Loss of Hippo signaling in liver activates YAP/TAZ, STAT3, Wnt/ $\beta$-catenin, and Notch signaling. YAP/TAZ forms a critical positive feedback loop with Notch signaling to promote liver enlargement and rapid HCC formation. Breaking this positive feedback loop leads to reduced hepatomegaly and tumor progression. The inhibitory role of $\mathrm{Wnt} / \beta$-catenin in the liver tumor caused by the vicious positive feedback loop occurs at least in part through the DP1-mediated inhibition of Notch signaling. STAT3 activation is dispensable for the development of HCC caused by loss of Hippo activity.

regeneration and survival of $\beta$-catenin-driven colon cancers (54, 76). However, we showed here that removal of $\beta$-catenin in the liver of DKO mice caused more severe liver enlargement and rapid HCC formation. Thus, the requirement of YAP and/or $\beta$-catenin for organ size control seems to depend on the tissue context. Similarly, Jag1 induction by YAP/TAZ and NICD interaction could also be tissue context dependent. While 2 TEAD response elements in the JAG1 promoter and the YAP/TEAD complex were found to be essential for JAG1 induction in HCC cells (49), YAP has also been found to require NICD to induce Jag1 expression in an RBP-Jא-dependent, but TEAD-independent, fashion in smooth muscle cells (50).

The roles of the Wnt/ $\beta$-catenin pathway in HCC also depend on genetic contexts. Although genetic mutations activating the Wnt/ $\beta$-catenin pathway have been characterized as a cause of various cancers including $\operatorname{HCC}(29,77,78)$, intriguingly, transgenic mice overexpressing either WT or constitutively active forms of $\beta$-catenin have failed to develop HCC. These results indicate that $\beta$-catenin alone is insufficient to initiate tumorigenesis in the liver. Instead, $\beta$-catenin may collaborate with other signaling pathways to contribute to HCC development. $\beta$-Catenin was reported to cooperate with activated H-Ras to induce HCC (79). It has also been reported that removal of $\beta$-catenin in hepatocytes resulted in a paradoxical increase in susceptibility to chemical-induced hepatocarcinogenesis (29). While it is possible that a decrease or disruption of Wnt/ $\beta$-catenin signaling further enhanced HCC in TKO$\beta$ cat mice through another mechanism(s) that is yet to be defined, the important clinical findings reported by Fitamant et al. that signatures of $\beta$-catenin activation and YAP activation are mutually exclusive in HCC human patients (80) strongly suggest that it is unlikely that Wnt/ $\beta$-catenin activation contributed to the development of HCC development in Hippo-DKO mice. In contrast, YAP and Notch activation signatures are highly correlated in HCC (80, 81), arguing for the clinical importance of the Notch and YAP/TAZ positive feedback loop we identified in this study. Therefore, inhibition of this positive feedback loop by Wnt/ $\beta$-catenin is at least one of the underlying mechanisms whereby HCC development in DKO mice was further enhanced upon $\beta$-catenin removal.

Another surprising finding of ours is that, in contrast to the discoveries in Drosophila, where loss of Hippo signaling promotes intestinal stem cell proliferation by activation of the JAK/ STAT pathway (66-68), STAT3 activation, which was probably induced by the IL- 6 family of cytokines from the newly infiltrated Kuppfer cells, was not required for the HCC formation caused by loss of Hippo signaling in mice, despite the fact that there is massive inflammation in the DKO liver (25). STAT3 activation was detected in a large number of human HCC specimens $(4,34,35)$, and hepatocyte-specific ablation of STAT3 in mice induced cell death and a significant reduction in mutagen-induced HCCs (34, 35). Our genetic studies indicate that activation of YAP/TAZ and Notch signaling is among the pathways that can potently promote HCC formation independently of STAT3 activation. As STAT3activating mutations are rare in human cancers, despite the finding that STAT3 activation is observed in approximately $60 \%$ of HCCs (34), our findings indicate that, depending on the primary genetic changes in HCC, inhibition of STAT3 or Wnt/ $\beta$-catenin may be ineffective or even deleterious.

HCC is the fifth most frequent malignant cancer and the second leading cause of cancer-related deaths worldwide. Our findings indicate that HCCs caused by different molecular lesions have to be treated using distinct therapeutic strategies, even though activation of common oncogenic pathways is observed in these distinct HCCs. Misregulation of the Hippo pathway has been reported at a high frequency in a variety of human cancers and correlates with poor patient prognosis. However, few somatic or germline mutations in Hippo pathway genes have been identified. Among Hippo signaling components, only the neurofibromin 2 (NF2) gene has been found to have cancer-causing mutations. Mst or Lats are well-defined tumor suppressors in mice, and epigenetic silencing of these genes that inhibits their kinase activity in cancer has been reported. In addition, the Hippo signaling downstream effector YAP1 has been reported to be overexpressed or amplified in various human and murine tumors. These findings suggest that the frequent disruption of Hippo signaling activity in human cancers results from molecular interaction with other events rather than somatic mutations of pathway genes. One scenario could be due to functional redundancy (loss-of-function mutations) or the weak effects of gain-of-function mutations in this pathway (82), since somatic mutations of many Hippo pathway genes are not enough to drive tumor formation. 
Our findings provide further mechanistic understandings to the recent clinical observations that human HCCs caused by Wnt/ $\beta$-catenin signaling activation and YAP/TAZ activation, respectively, have distinct features and are mutually exclusive (80). In addition, recent work suggests that Notch activation inversely correlates with $\beta$-catenin signaling in patients with HCC (81). Our study highlights the necessity for the accurate classification of HCCs according to their cellular and molecular signatures in order to develop effective therapeutic strategies that target distinct HCC subgroups. Inhibition of $\beta$-catenin may be beneficial in HCCs primarily caused by mutations that activate $\beta$-catenin, but would be detrimental to HCCs primarily caused by mutations that activate YAP/TAZ or Notch signaling.

\section{Methods}

Mouse lines and drug treatment. The Alb-Cre Mst1 ${ }^{-/-} \mathrm{Mst} 2^{f / / l l}$ mouse lines have been described previously (25). Both male and female mice were used, because they did not show differences in this study. To generate loss of $\beta$-catenin, Stat3, or Jag1 in DKO mice, we crossed Alb-Cre Mst1-1$M s t 2^{f / f l}$ mice with Ctnnb $1^{f / f l}$, Stat $3^{f / f}$ (provided by Xin-Yuan Fu, Indiana University School of Medicine, Indianapolis, Indiana, USA), or Jag ${ }^{1 / / f A}$ mice (Jag1 $1^{\text {tmlGrid }}$; The Jackson Laboratory), respectively. Mice were treated with DAPT (Calbiochem); iCRT3 (Sigma-Aldrich); or Stattic (ApexBio Technology) by i.p. injection at a concentration of $125 \mathrm{mg} / \mathrm{kg}$ or 20 $\mathrm{mg} / \mathrm{kg}$, respectively, in DMSO (Sigma-Aldrich), every other day or in equivalent volumes of vehicle as a control. Mice received 10 injections during the 3-week period starting at 2 months (DKO) or 1 month (TKO$\beta$-cat) of age for DAPT. One week later, mice were given ten more injections over a three-week period, which completed the treatment (total of 20 injections). For iCRT3 and Stattic, DKO mice received 10 injections over a 3-week period, starting at 1 month of age. One week later, mice were given ten more injections over a three-week period, which completed the treatment (total of 20 injections).

Hepatocyte isolation. Hepatocyte isolation was performed via collagenase-elastase perfusion according to the manufacturer's protocol (Worthington Biochemical). Briefly, mice were anesthetized with sodium pentobarbital solution (30 $\mathrm{mg} / \mathrm{kg}$ i.p.) and their livers perfused with calcium- and magnesium-free HBSS (CMF-HBSS), followed by enzyme buffer solution $(225 \mathrm{U} / \mathrm{ml}$ collagenase, $0.3 \mathrm{U} / \mathrm{ml}$ elastase, and $10 \mathrm{U} / \mathrm{ml}$ DNase). The isolated mouse hepatocytes were then cultured in DMEM containing 10\% FBS, L-glutamin, and penicillin-streptomycin in collagen-coated plates (Biocoat; BD).

Cell culture, siRNA knockdown, and transfections. All cell lines were maintained in DMEM (Gibco, Thermo Fisher Scientific) supplemented with 10\% FBS, L-glutamine (Gibco, Thermo Fisher Scientific), and 100 $\mathrm{U} / \mathrm{ml}$ penicillin-streptomycin (Gibco, Thermo Fisher Scientific). siRNA transfections were performed with Lipofectamine RNAiMAX Reagent (Life Technologies, Thermo Fisher Scientific) in antibiotic-free medium according to the manufacturer's instructions. The following oligonucleotides of siRNA duplexes (Life Technologies, Thermo Fisher Scientific) were used: sigfP, 5'-GTTCAGCGTGTCCGGCGAG-3'; siYAP, 5'-GACATCTTCTGGTCAGAGA-3; siTAZ, 5'-ACGTTGACTTAGGAACTTT-3'; siDP1\#1, 5'-GGAGACTTGAAAGAATAAA-3'; and siDP1\#2, 5'-GGACCACTTCCTACAACGA-3'. Plasmid transfections were done using Lipofectamine 2000 and 3000 (Life Technologies, Thermo Fisher Scientific) or TransIT-LT1 (Mirus Bio) according to the manufacturers' instructions.
IHC. Liver tissues were perfused with PBS and fixed in 4\% paraformaldehyde for preparation of frozen sections and paraffin-embedded sections. Sections were stained with H\&E or by IHC according to standard procedures. For ABC staining, tissues were deparaffinized, and antigen retrieval was performed according to the manufacturer's instructions (IHC-101; Bethyl Laboratories). Sections were blocked in rabbit serum, followed by incubation for 2 hours with the following primary antibodies: $\beta$-catenin (1:100; Cell Signaling Technology; catalog 9502); YAP/TAZ (1:50; Cell Signaling Technology; catalog 8418); and NICD (1:25; Abcam; catalog ab52301). TUNEL tissue staining was performed using a modified protocol for the ApopTag Plus In Situ Apoptosis Fluorescein Detection Kit (EMD Millipore; catalog S7111). Briefly, liver tissues were deparaffinized and then treated with proteinase K (20pTag) for 15 minutes at room temperature, followed by 3 washes in PBS. Specimens were applied to equilibration buffer for 30 seconds at room temperature and then incubated with TdT enzyme in a humidified chamber at $37^{\circ} \mathrm{C}$ for 1 hour. The enzyme reaction was stopped with Stop/Wash buffer for 10 minutes at room temperature, followed by 3 washes in PBS. Tissues were incubated in the dark with antidigoxigenin conjugate in a humidified chamber for 30 minutes. Specimens were then washed 4 times in PBS. Mounting medium containing DAPI was applied to the specimen and then viewed by fluorescence microscopy (Zeiss 510 NLO Meta) using the appropriate excitation and emission filters.

Vectors. Constructs encoding YAP or TAZ were generated by subcloning the PCR products of a cDNA encoding human YAP or human TAZ from HEK293T cells in pCMV4-Flag or pEGFP-C1 vectors. DP1 expression constructs were constructed as previously described (61). p3XFlag-CMV7-NICD1 (plasmid 20183); p3XFlag-CMV7-NICD2 (plasmid 20184); p3XFlag-CMV7-NICD3 (plasmid 20185); p3XFlagCMV7-NICD4 (plasmid 20186); pcDNA3- $\gamma$-catenin (plasmid 16827); and pcDNA3-HA-TAZ (plasmid 32839) were purchased from Addgene. NICD $\triangle \mathrm{PEST}$ ( $\triangle$ amino acid 2443-2555) was generated from WT NICD. Constructs encoding various TAZ mutants were generated by PCR subcloning of the cDNA human TAZ from the pcDNA3-HA vector described above. 6xNRE- and 3xSd-lucirease constructs were provided by Tohru Ishitani (Kyusu University, Fukuoka, Japan) and Jin Jiang (University of Texas Southwestern Medical Center, Dallas, Texas, USA), respectively.

Immunoprecipitation and immunoblotting. Cells or mouse liver tissues were lysed in lysis buffer (20 mM Tris [pH 7.4], $150 \mathrm{mM} \mathrm{NaCl}, 1 \%$ Triton X-100, 1 mM EDTA, 1 mM EGTA, 2.5 mM sodium pyrophosphate, $1 \mathrm{mM} \beta$-glycerophosphate, $1 \mathrm{mM}$ sodium orthovanadate) or radioimmunoprecipitation assay (RIPA) buffer (Santa Cruz Biotechnology Inc.; catalog sc-24948), respectively, containing protease inhibitor cocktail (Roche). Lysates were clarified by centrifugation at $18,500 \mathrm{~g}$ for 10 minutes at $4^{\circ} \mathrm{C}$, and protein concentration was determined using a Bradford Assay Kit (Bio-Rad). Proteins were immunoprecipitated from cell lysates with the indicated antibodies and Protein A/G Plus-Agarose Beads (Santa Cruz Biotechnology Inc.; catalog sc-2003). Immnoprecipitates (500-800 $\mu \mathrm{g})$ and total cell lysates $(20-50 \mu \mathrm{g})$ were analyzed by Western blotting. The boiled samples were separated on Bis-Tris protein gels and transferred to nitrocellulose membranes (Life Technologies, Thermo Fisher Scientific). After blocking with 5\% nonfat skim milk or BSA in TBS with $0.1 \%$ Tween-20 for 1 hour, the membranes were probed with the corresponding antibodies overnight. Bound antibodies were visualized by ECL (EMD Millipore or Pierce, Thermo Fisher Scientific) using HRP-conjugated antibodies. 
Antibodies. Anti-YAP (catalog 4912); anti-p-YAP (catalog 4911); anti-JAG1 (catalog 2620); anti-cleaved Notch 1 (Val1744, catalogs 4147 and 2421); anti-p-STAT3 (catalog 9145), anti-MST1 (catalog 3682); anti-MST2 (catalog 3952); anti-LATS1 (catalog 9153); anti-pLATS1 (catalog Thr1079, 8654); and anti-LATS2 (catalog 5888) were purchased from Cell Signaling Technology. Anti-TAZ (catalog 560235 ) and anti- $\beta$-catenin (catalog 610153) antibodies were from BD Transduction Laboratories. Anti- $\beta$-TrCP (catalog 373400) antibody was purchased from Life Technologies (Thermo Fisher Scientific). Anti-cleaved Notch 1 (Val1744, catalog ab52301); anti-HES1 (catalog ab71559); and anti-STAT3 (catalog ab7966) antibodies were obtained from Abcam. Anti-Flag (clone M2, catalog F3165); anti-GAPDH (catalog SAB1405848); and anti-TAZ (catalog T4077) antibodies were purchased from Sigma-Aldrich. Anti-MST2 (catalog 1943-1) and antiSOX9 (catalog AB5535) antibodies were from Epitomics and EMD Millipore, respectively. Anti-YAP (clone H-125, catalog sc-15407); antiGFP (clone B-2; catalog sc9996); anti-HA (clone F-7, catalog sc-7392); anti-TAZ (clone H-70, catalog sc-48805); anti-DP1 (clone K-20, catalog sc-610); anti- $\gamma$-catenin (clone C-20, catalog sc-1497); and anti-STAT3 (clone C-20, catalog sc-482) antibodies were from Santa Cruz Biotechnology Inc. Anti-HA (catalog 11867423001) and DAPI were purchased from Roche and Vector Laboratories, respectively. Anti-JAG1-neutralizing antibody (AF1277 for human and AF599 for mouse) was purchased from R\&D Systems. Anti-mouse and rabbit HRP-conjugated secondary antibodies were from GE Healthcare Life Sciences. Anti-goat and anti-rat HRP-conjugated secondary antibodies were from Santa Cruz Biotechnology Inc. and Sigma-Aldrich, respectively.

Immunofluorescence. Liver tissues were perfused with PBS and fixed in $4 \%$ paraformaldehyde for the preparation of frozen sections and paraffin-embedded sections. Sections were permeabilized in 0.5\% Triton/ PBS (PBST) for 5 minutes, followed by 3 washes in PBS. Sections were incubated in blocking buffer (1\% BSA in PBST) for 1 hour at room temperature and then incubated overnight at $4^{\circ} \mathrm{C}$ with the primary antibody indicated in the blocking buffer. The antibodies for immunostaining included: pH3 (catalog 9701; Cell Signaling Technology); Sox9 (catalog ab5535; EMD Millipore); EPCAM (clone G8.8; Developmental Studies Hybridoma Bank, University of Iowa, Iowa City, Iowa, USA); DP1 (catalog sc-610; Santa Cruz Biotechnology Inc.); $\gamma$-catenin (catalog sc-1497; Santa Cruz Biotechnology); and F4/80 (catalog MCA497; AbD Serotec). Sections were washed 5 times in PBS and then incubated with a secondary antibody for 1 hour at room temperature. Donkey anti-mouse Alexa Fluor 488, donkey anti-rat Alexa Fluor 594, or donkey anti-rabbit Alexa Fluor 594 secondary antibodies (Life Technologies, Thermo Fisher Scientific) were used. Sections were washed 10 times in PBST and then mounted with mounting medium with DAPI (catalog H-1200; Vector Laboratories). Immunofluorescence imaging was performed using a Zeiss 510 NLO META laser scanning microscope.

Flow cytometric analysis. After incubation with Fc-blocker (2.4G2; $\mathrm{BD}$ Biosciences) for 10 minutes at room temperature, cells were stained with the following antibodies from eBioscience: phycoerythrin-conjugated (PE-conjugated) anti-CD11b (clone M1/70, catalog 14-0112) and allophycocyanin-conjugated (APC-conjugated) F4/80 (clone BM8, catalog 17-4801). Flow cytometric analysis was performed on a BD LSR II using FlowJo software.

$q R T-P C R$. Total RNA from mouse liver tissue or cultured cells was prepared using TRIzol Reagent (Life Technologies, Thermo Fisher Scientific) or an RNeasy Mini Kit (QIAGEN) according to the manu- facturer's protocol. cDNA were synthesized from total RNA (1-3 $\mu \mathrm{g})$ using SuperScript II Reverse Transcriptase with a random primer (Life Technologies, Thermo Fisher Scientific). qRT-PCR was performed using SYBR Select Master Mix (Thermo Fisher Scientific) on a StepOnePlus thermal cycler from Applied Biosystems. Expression levels were always given relative to GAPDH.

The following PCR primers for mouse samples were used: Ctgf, forward, CTGCCTACCGACTGGAAGAC; reverse, CATTGGTAACTCGGGTGGAG; Cyr61, forward, GCTCAGTCAGAAGGCAGACC, reverse, GTTCTTGGGGACACAGAGGA; Axin2, forward, GCTGGAGAAACTGAAACTGGA, reverse, CAAAGTGTTGGGTGGGGTAAG; Wifl, forward, GCCACGAACCCAACAAGT, reverse, TCCCTTCTATCCTCAGCСTTT; Apcdd1, forward, ATGAACACCACCСTCCCATAC, reverse, GTAGTAATGCCCTTCCCAGGT; Nrarp, forward, GCGTGGTTATGGGAGAAAGAT, reverse, GGGAGAGGAAAAGAGGAATGA; Hes1, forward, GTGGGTCCTAACGCAGTGTC, reverse, TCAGAAGAGAGAGGTGGGCTA; Jag1, forward, AGAAGTCAGAGTTCAGAGGCGTCC, reverse, AGTAGAAGGCTGTCACCAAGCAAC; Jag2, forward, AGCCACGGAGCAGTCATTTG, reverse, TCGGATTCCAGAGCAGATAGCG; Il6, forward, TCCATCCAGTTGCCTTCTTG, reverse, TTCCACGATTTCCCAGAGAA; Il11, forward, AGGCGAGACATCAAGAGCTG, reverse, GCAGGTGGTCCTTCCCTAA; Lif, forward, ATTGTGCCCTTACTGCTGCTG, reverse, GCCAGTTGATTCTTGATCTGGT;Osm, forward,CCCTATATCCGCCTCCAAAACC, reverse, GACTCTGTCCAGTGTGGTGTAC; Illb, forward, CAACCAACACGTGATATTCTCCATG, reverse, GATCCACACTCTCCAGCTGCA; Il33, forward, TCCTTGCTTGGCAGTATCCA, reverse, TGCTCAATGTGTCAACAGACG; Ccl4, forward, GCCСTCTCTCTCCTCTTGCT, reverse, CTGGTCTCATAGTAATCCATC; Cxcl10, forward, GTCACATCAGCTGCTACTC, reverse, GTGGTTAAGTTCGTGCTTAC; Cxcl16, forward, GGGAAGAGTTTTCACCACCA, reverse, GGTTGGGTGTGCTCTTTGTT; Bcl-2, forward, GGACTTGAAGTGCCATTGGT, reverse, AGCCCCTCTGTGACAGCTTA; Socs3, forward, AGCTTACTACATCTATTCT, reverse, TTAAAGTGGAGCATCATACT; EpCam, forward, CCTGAGAGTGAACGGAGAGC, reverse, GACACCACCACAATGACAGC; Sox9, forward, CGACTACGCTGACCATCAGA, reverse, AGACTGGTTGTTCCCAGTGC; Gapdh, forward, ATCCTGCACCACCAACTGCT, reverse, GGGCCATCCACAGTCTTCTG.

The following primers for human samples were used: $C T G F$, forward, AGGAGTGGGTGTGTGACGA, reverse, CCAGGCAGTTGGCTCTAATC; ANKRD1, forward, AGTAGAGGAACTGGTCACTGG, reverse, TGGGCTAGAAGTGTCTTCAGAT; HES1, forward, GGCTGGAGAGGCGGCTAA, reverse, GAGAGGTGGGTTGGGGAGTT; HEY2, forward, GTACCATCCAGCAGTGCATC, reverse, AGAGAATTCAGTCAGGGCATTT; JAG1, forward, CAACCGTGCCAGTGACTATTTCTGC, reverse, TGTTCCCGTGAAGCCTTTGTTACAG; JAG2, forward, AACGATACCCCGAATGAGG, reverse, GCTGCCACAGTAGTTCAGGTCTTTG; TAZ, forward, GGCTGGGAGATGACCTTCAC, reverse, CTGAGTGGGGTGGTTCTGCT; GAPDH, forward, AGCCACATCGCTCAGACAC, reverse, GCCCAATACGACCAAATCC.

Luciferase reporter assays. To measure the activity of YAP/TAZ, Wnt/ $\beta$ catenin, and Notch signaling, cells seeded in 24 -well plates were cotransfected with a Scalloped-dependent (Sc-dependent) luciferase reporter, 3XSd-Luc, a 8XSuper Top-Flash or Notch-responsive luciferase reporter, 6XNRE-Luc, respectively, with pTK-Renilla (Promega) and effector plasmids. Luciferase activity was measured with a dualluciferase reporter assay kit (Promega) according to the manufacturer's 
instructions. Renilla activity was used to normalize luciferase reporter activity. For knockdown experiments, cells were transfected with 20 nM siRNA for 48 hours before plasmid transfection.

Statistics. All data analysis in this study was carried out using Microsoft Excel or GraphPad Prism 7 (GraphPad Software) for Mac OS. Quantifications were done from at least 3 independent experimental groups. Statistical analysis between groups was performed by 2-tailed Student's $t$ test to determine significance when only 2 groups were compared. Oneway ANOVA with Tukey's post-hoc tests were used to compare differences between multiple groups. $P$ values of less than 0.05 and 0.01 were considered significant. Error bars on all graphs are presented as the SEM of the mean unless otherwise indicated. Differences in Kaplan-Meier survival curves were analyzed using the long-rank statistic.

Study approval. The procedures for all animal experiments were reviewed and approved by the IACUCs of the NIH and Harvard Medical School.

\section{Author contributions}

WK, SKK, YK, EHJ, BG and YY designed the experiments. WK, SKK, JGJ, YK, JD, HK, and OP performed genetic crosses, tissue sampling, and experiments. TI performed ISH using zebrafish embryos. WK, SKK, JGJ, YK, EHJ, TI, and YY interpreted the data. WK, EHJ, BG, and YY wrote and reviewed the manuscript. YY supervised the project.

\section{Acknowledgments}

We thank members of the Yang laboratory for stimulating discussions and J. Jiang for reagents (University of Texas Southwestern Medical Center, Dallas, Texas). This study was supported by grants from the intramural research program of the NHGRI and the HSDM and by a Korean Visiting Scientist Training Award (KVSTA) fellowship from the Korean Health Industry Development Institute (KHIDI) (HI13C1274, to WK and HI13C1276, to HK). This research was partially supported by the National R\&D Program for Cancer Control of the Ministry of Health and Welfare of Korea (1420060, to EHJ).

Address correspondence to: Yingzi Yang, Department of Developmental Biology, Harvard School of Dental Medicine, 188 Longwood Avenue, Boston, Massachusetts 02115, USA. Phone:617.432.8304; E-mail: yingzi_yang@hsdm.harvard.edu.
1. Farazi PA, DePinho RA. Hepatocellular carcinoma pathogenesis: from genes to environment. Nat Rev Cancer. 2006;6(9):674-687.

2. Hanahan D, Weinberg RA. Hallmarks of cancer: the next generation. Cell. 2011;144(5):646-674.

3. Forner A, Llovet JM, Bruix J. Hepatocellular carcinoma. Lancet. 2012;379(9822):1245-1255.

4. Yu H, Lee H, Herrmann A, Buettner R, Jove R. Revisiting STAT3 signalling in cancer: new and unexpected biological functions. Nat Rev Cancer. 2014;14(11):736-746.

5. Miyajima A, Tanaka M, Itoh T. Stem/progenitor cells in liver development, homeostasis, regeneration, and reprogramming. Cell Stem Cell. 2014;14(5):561-574.

6. El-Serag HB. Hepatocellular carcinoma. N Engl J Med. 2011;365(12):1118-1127.

7. Torre LA, Bray F, Siegel RL, Ferlay J, Lortet-Tieulent J, Jemal A. Global cancer statistics, 2012. CA Cancer J Clin. 2015;65(2):87-108.

8. Yimlamai D, Fowl BH, Camargo FD. Emerging evidence on the role of the Hippo/YAP pathway in liver physiology and cancer. J Hepatol. 2015;63(6):1491-1501.

9. Dong J, et al. Elucidation of a universal size-control mechanism in Drosophila and mammals. Cell. 2007;130(6):1120-1133.

10. Huang J, Wu S, Barrera J, Matthews K, Pan D. The Hippo signaling pathway coordinately regulates cell proliferation and apoptosis by inactivating Yorkie, the Drosophila Homolog of YAP. Cell. 2005;122(3):421-434.

11. Wu S, Huang J, Dong J, Pan D. hippo encodes a Ste20 family protein kinase that restricts cell proliferation and promotes apoptosis in conjunction with salvador and warts. Cell. 2003;114(4):445-456.

12. Chan EH, Nousiainen M, Chalamalasetty RB, Schäfer A, Nigg EA, Silljé HH. The Ste20like kinase Mst2 activates the human large tumor suppressor kinase Lats1. Oncogene. 2005;24(12):2076-2086.

13. Yu FX, et al. Regulation of the Hippo-YAP path- way by G-protein-coupled receptor signaling. Cell. 2012;150(4):780-791.

14. Aragona M, et al. A mechanical checkpoint controls multicellular growth through YAP/ TAZ regulation by actin-processing factors. Cell. 2013;154(5):1047-1059.

15. Dupont S, et al. Role of YAP/TAZ in mechanotransduction. Nature. 2011;474(7350):179-183.

16. Halder G, Dupont S, Piccolo S. Transduction of mechanical and cytoskeletal cues by YAP and TAZ. Nat Rev Mol Cell Biol. 2012;13(9):591-600.

17. Zhao B, et al. Inactivation of YAP oncoprotein by the Hippo pathway is involved in cell contact inhibition and tissue growth control. Genes Dev. 2007;21(21):2747-2761.

18. Zhao B, Li L, Tumaneng K, Wang CY, Guan KL. A coordinated phosphorylation by Lats and CK1 regulates YAP stability through SCF(beta-TRCP). Genes Dev. 2010;24(1):72-85.

19. Lei QY, et al. TAZ promotes cell proliferation and epithelial-mesenchymal transition and is inhibited by the hippo pathway. Mol Cell Biol. 2008;28(7):2426-2436.

20. Harvey KF, Zhang X, Thomas DM. The Hippo pathway and human cancer. Nat Rev Cancer. 2013;13(4):246-257.

21. Zhou D, et al. Mst1 and Mst2 protein kinases restrain intestinal stem cell proliferation and colonic tumorigenesis by inhibition of Yes-associated protein (Yap) overabundance. Proc Natl Acad Sci U S A. 2011;108(49):E1312-E1320.

22. Zhang $X$, et al. The Hippo pathway transcriptional co-activator, YAP, is an ovarian cancer oncogene. Oncogene. 2011;30(25):2810-2822.

23. Cordenonsi M, et al. The Hippo transducer TAZ confers cancer stem cell-related traits on breast cancer cells. Cell. 2011;147(4):759-772.

24. Lee KP, et al. The Hippo-Salvador pathway restrains hepatic oval cell proliferation, liver size, and liver tumorigenesis. Proc Natl Acad Sci U S A. 2010;107(18):8248-8253.

25. Song H, et al. Mammalian Mst1 and Mst2 kinases play essential roles in organ size control and tumor suppression. Proc Natl Acad Sci U S A. 2010;107(4):1431-1436.

26. Clevers $H$, Nusse R. Wnt/ $\beta$-catenin signaling and disease. Cell. 2012;149(6):1192-1205.

27. de La Coste A, et al. Somatic mutations of the beta-catenin gene are frequent in mouse and human hepatocellular carcinomas. Proc Natl Acad Sci U S A. 1998;95(15):8847-8851.

28. Feng GS. Conflicting roles of molecules in hepatocarcinogenesis: paradigm or paradox. Cancer Cell. 2012;21(2):150-154.

29. Nejak-Bowen KN, et al. Accelerated liver regeneration and hepatocarcinogenesis in mice overexpressing serine- 45 mutant beta-catenin. Hepatology. 2010;51(5):1603-1613.

30. Feng GJ, et al. Conditional disruption of Axin1 leads to development of liver tumors in mice. Gastroenterology. 2012;143(6):1650-1659.

31. Rajewsky MF, Dauber W, Frankenberg H. Liver carcinogenesis by diethylnitrosamine in the rat. Science. 1966;152(3718):83-85.

32. Jhappan C, Stahle C, Harkins RN, Fausto N, Smith GH, Merlino GT. TGF alpha overexpression in transgenic mice induces liver neoplasia and abnormal development of the mammary gland and pancreas. Cell. 1990;61(6):1137-1146.

33. Conner EA, Lemmer ER, Omori M, Wirth PJ, Factor VM, Thorgeirsson SS. Dual functions of E2F-1 in a transgenic mouse model of liver carcinogenesis. Oncogene. 2000;19(44):5054-5062.

34. He G, et al. Hepatocyte IKKbeta/NF-kappaB inhibits tumor promotion and progression by preventing oxidative stress-driven STAT3 activation. Cancer Cell. 2010;17(3):286-297.

35. He G, Karin M. NF-кB and STAT3 - key players in liver inflammation and cancer. Cell Res. 2011;21(1):159-168.

36. Villanueva A, et al. Notch signaling is activated in human hepatocellular carcinoma and induces tumor formation in mice. Gastroenterology. 2012;143(6):1660-1669.e7. 
37. Zong Y, et al. Notch signaling controls liver development by regulating biliary differentiation. Development. 2009;136(10):1727-1739.

38. Geisler F, Strazzabosco M. Emerging roles of Notch signaling in liver disease. Hepatology. 2015;61(1):382-392.

39. $\mathrm{Lu} \mathrm{L}$, et al. Hippo signaling is a potent in vivo growth and tumor suppressor pathway in the mammalian liver. Proc Natl Acad Sci U S A. 2010;107(4):1437-1442.

40. Zhou D, et al. Mst1 and Mst2 maintain hepatocyte quiescence and suppress hepatocellular carcinoma development through inactivation of the Yap1 oncogene. Cancer Cell. 2009;16(5):425-438.

41. Antoniou A, et al. Intrahepatic bile ducts develop according to a new mode of tubulogenesis regulated by the transcription factor SOX9. Gastroenterology. 2009;136(7):2325-2333.

42. Tanimizu N, Mitaka T. Re-evaluation of liver stem/progenitor cells. Organogenesis. 2014;10(2):208-215

43. Morell CM, Strazzabosco M. Notch signaling and new therapeutic options in liver disease. J Hepatol. 2014;60(4):885-890.

44. Yimlamai D, et al. Hippo pathway activity influences liver cell fate. Cell. 2014;157(6):1324-1338.

45. Struhl G, Fitzgerald K, Greenwald I. Intrinsic activity of the Lin-12 and Notch intracellular domains in vivo. Cell. 1993;74(2):331-345

46. Kopan R, Ilagan MX. The canonical Notch signaling pathway: unfolding the activation mechanism. Cell. 2009;137(2):216-233.

47. Fortini ME. Notch signaling: the core pathway and its posttranslational regulation. Dev Cell. 2009;16(5):633-647.

48. Schroeter EH, Kisslinger JA, Kopan R. Notch-1 signalling requires ligand-induced proteolytic release of intracellular domain. Nature. 1998;393(6683):382-386.

49. Tschaharganeh DF, et al. Yes-associated protein up-regulates Jagged-1 and activates the Notch pathway in human hepatocellular carcinoma. Gastroenterology. 2013;144(7):1530-1542.e12.

50. Manderfield LJ, et al. Hippo signaling is required for Notch-dependent smooth muscle differentiation of neural crest. Development. 2015;142(17):2962-2971.

51. Andersson ER, Lendahl U. Therapeutic modulation of Notch signalling--are we there yet? Nat Rev Drug Discov. 2014;13(5):357-378.

52. Liu CY, et al. The hippo tumor pathway promotes TAZ degradation by phosphorylating a phosphodegron and recruiting the SCF $\{$ beta\}-TrCP E3 ligase. J Biol Chem. 2010;285(48):37159-37169.

53. Heallen T, et al. Hippo pathway inhibits Wnt signaling to restrain cardiomyocyte proliferation and heart size. Science. 2011;332(6028):458-461.

54 . Rosenbluh J, et al. $\beta$-Catenin-driven cancers require a YAP1 transcriptional complex for survival and tumorigenesis. Cell. 2012;151(7):1457-1473.

55. Guo X, Day TF, Jiang X, Garrett-Beal L, Topol L, Yang Y. Wnt/beta-catenin signaling is sufficient and necessary for synovial joint formation. Genes Dev. 2004;18(19):2404-2417.

56. Wickline ED, Awuah PK, Behari J, Ross M, Stolz DB, Monga SP. Hepatocyte $\gamma$-catenin compensates for conditionally deleted $\beta$-catenin at adherens junctions. J Hepatol. 2011;55(6):1256-1262.

57. Wickline ED, Du Y, Stolz DB, Kahn M, Monga SP. $\gamma$-Catenin at adherens junctions: mechanism and biologic implications in hepatocellular cancer after $\beta$-catenin knockdown. Neoplasia. 2013;15(4):421-434.

58. Gonsalves FC, et al. An RNAi-based chemical genetic screen identifies three small-molecule inhibitors of the Wnt/wingless signaling pathway. Proc Natl Acad Sci U S A. 2011;108(15):5954-5963.

59. Girling R, et al. A new component of the transcription factor DRTF1/E2F. Nature. 1993;362(6415):83-87.

60. van den Heuvel S, Dyson NJ. Conserved functions of the pRB and E2F families. Nat Rev Mol Cell Biol. 2008;9(9):713-724.

61. Kim WT, et al. Dual functions of DP1 promote biphasic Wnt-on and Wnt-off states during anteroposterior neural patterning. ЕMBO J. 2012;31(16):3384-3397.

62. Hübscher SG. Histological assessment of non-alcoholic fatty liver disease. Histopathology. 2006;49(5):450-465.

63. Davies LC, Jenkins SJ, Allen JE, Taylor PR. Tissue-resident macrophages. Nat Immunol. 2013;14(10):986-995.

64. Taub R. Liver regeneration: from myth to mechanism. Nat Rev Mol Cell Biol. 2004;5(10):836-847.

65. Yu H, Pardoll D, Jove R. STATs in cancer inflammation and immunity: a leading role for STAT3. Nat Rev Cancer. 2009;9(11):798-809.

66. Shaw RL, Kohlmaier A, Polesello C, Veelken C, Edgar BA, Tapon N. The Hippo pathway regulates intestinal stem cell proliferation during Drosophila adult midgut regeneration. Development. 2010;137(24):4147-4158.

67. Staley BK, Irvine KD. Warts and Yorkie mediate intestinal regeneration by influencing stem cell proliferation. Curr Biol. 2010;20(17):1580-1587.
68. Ren F, Wang B, Yue T, Yun EY, Ip YT, Jiang J. Hippo signaling regulates Drosophila intestine stem cell proliferation through multiple pathways. Proc Natl Acad Sci U S A. 2010;107(49):21064-21069.

69. Schust J, Sperl B, Hollis A, Mayer TU, Berg T. Stattic: a small-molecule inhibitor of STAT3 activation and dimerization. Chem Biol. 2006;13(11):1235-1242.

70. Si-Tayeb K, Lemaigre FP, Duncan SA. Organogenesis and development of the liver. Dev Cell. 2010;18(2):175-189.

71. Cai J, Maitra A, Anders RA, Taketo MM, Pan D. $\beta$-Catenin destruction complex-independent regulation of Hippo-YAP signaling by APC in intestinal tumorigenesis. Genes Dev. 2015;29(14):1493-1506.

72. Park HW, et al. Alternative Wnt Signaling Activates YAP/TAZ. Cell. 2015;162(4):780-794.

73. Torre C, Perret C, Colnot S. Molecular determinants of liver zonation. Prog Mol Biol Transl Sci. 2010;97:127-150.

74. Boulter L, et al. Macrophage-derived Wnt opposes Notch signaling to specify hepatic progenitor cell fate in chronic liver disease. Nat Med. 2012;18(4):572-579.

75. Azzolin L, et al. Role of TAZ as mediator of Wnt signaling. Cell. 2012;151(7):1443-1456.

76. Oudhoff MJ, et al. SETD7 Controls Intestinal Regeneration and Tumorigenesis by Regulating Wnt/ $\beta$-Catenin and Hippo/YAP Signaling. Dev Cell. 2016;37(1):47-57.

77. Tan X, et al. Epidermal growth factor receptor: a novel target of the Wnt/beta-catenin pathway in liver. Gastroenterology. 2005;129(1):285-302.

78. Cadoret A, et al. Hepatomegaly in transgenic mice expressing an oncogenic form of betacatenin. Cancer Res. 2001;61(8):3245-3249.

79. Harada N, Oshima H, Katoh M, Tamai Y, Oshima M, Taketo MM. Hepatocarcinogenesis in mice with beta-catenin and Ha-ras gene mutations. Cancer Res. 2004;64(1):48-54.

80. Fitamant J, et al. YAP Inhibition Restores Hepatocyte Differentiation in Advanced HCC, Leading to Tumor Regression. Cell Rep. 2015;10(10):1692-1707.

81. Zhang Q, et al. Notch3 functions as a regulator of cell self-renewal by interacting with the $\beta$-catenin pathway in hepatocellular carcinoma. Oncotarget. 2015;6(6):3669-3679.

82. Chen Q, et al. Homeostatic control of Hippo signaling activity revealed by an endogenous activating mutation in YAP. Genes Dev. 2015;29(12):1285-1297. 